Pacific Journal of Mathematics

QUOTIENTS OF THE COMPLEX BALL BY DISCRETE 


\title{
QUOTIENTS OF THE COMPLEX BALL BY DISCRETE GROUPS
}

\author{
F. C. Kirwan, R. Lee, and S. H. Weintraub
}

In this paper we systematically study varieties $Q(\mu)$, which are compactifications of the space $Q$ of distinct points in $\left(P^{\overline{1}}\right)^{r}$ given by a sequence of "weights" $\underline{\mu}$, and which for certain $\underline{\mu}$ are also compactification of the quotient of the complex $r$-ball by discrete subgroups $\Gamma(\underline{\mu})$ of $\mathrm{PU}(r, 1)$, as discovered by Deligne and Mostow.

We obtain a wealth of topological information about the spaces $Q(\mu)$ and their desingularizations $Q^{*}(\mu)$. In some cases we can completely describe them. Otherwise, we obtain computations of Betti numbers and Hodge numbers. As applications we determine the $L^{2}$-cohomology and in many cases the (ordinary) rational cohomology of the groups $\Gamma(\underline{\mu})$.

0. Introduction. In this paper we study a family of algebraic varieties which arise in two ways. The first is as quotients of the ball in $\mathbf{C}^{r}$ by discrete subgroups of $\mathrm{PU}(r, 1)$, and the second as various compactifications of the configuration space $Q$ of $r$ distinct points in $\mathbf{P}^{1}$.

They were first discovered by Deligne and Mostow ([DM], $[\mathbf{M}])$ where they arose through the investigation of generalized hypergeometric functions. We briefly recapitulate their work in $\$ 2$ below.

We wish to study those varieties systematically. In this connection, we find that the second viewpoint, in terms of Mumford's geometric invariant theory [Mu], is more useful. Let $N=r+3$ and let $\mu=\left(\mu_{1}, \ldots, \mu_{N}\right)$ be a sequence of positive integers (which we call "weights"). Associated to $\mu$ is a line bundle over $\left(\mathbf{P}^{1}\right)^{N}$, and hence an $r$-dimensional projective variety $Q(\underline{\mu})$ obtained by taking the semistable points with respect to the linear action of $\mathrm{PGL}_{2}$ and then forming the quotient space in the sense of geometric invariant theory (see $\S 6$ ). $Q(\mu)$ is a compactification of $Q$, and the varieties of Deligne and Mostow arise as $Q(\underline{\mu})$ for $\underline{\mu}$ satisfying certain arithmetical conditions.

These varieties $Q(\mu)$ are always rational (1.11). When $r=2$, and when $r=3$ and $Q(\underline{\mu})$ is nonsingular, they can be completely described (4.1). (When $r=2$ the possibilities for $Q(\underline{\mu})$ are $\mathbf{P}^{1} \times \mathbf{P}^{1}$ or $\mathbf{P}^{2}$ with $k$ points blown up, $0 \leq k \leq 4$. When $r=\overline{3}$ the possibilities are more complicated.) In this case our work also determines the rational cohomology of the associated discrete subgroup $\Gamma(\underline{\mu})$ of $\mathrm{PU}(r, 1)$. 
In general $Q(\underline{\mu})$ is singular, and has a desingularization $Q^{*}(\underline{\mu})$ obtained by blowing up its singular "cusps" (1.10). The basic topological invariants of these varieties $Q^{*}(\underline{\mu})$ are their Betti numbers and Hodge numbers, for which we have a complete analysis (7.14). One case of

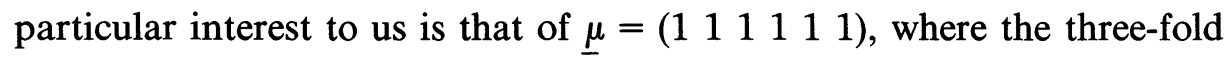
$Q^{*}(\underline{\mu})$ coincides with the moduli space of curves studied extensively in $\left[\mathbf{L} \mathbf{W}_{\mathbf{1}}\right],\left[\mathbf{L} \mathbf{W}_{\mathbf{2}}\right]$.

For the singular varieties $Q(\mu)$, it is natural to compute their intersection homology, which we do in (8.6). Since these singular varieties can be identified with Baily-Borel-Satake compactifications of the space $Q$ their intersection homology is the same as $L^{2}$-cohomology. Thus our results give computations of $L^{2}$-cohomology, and as far as we know the first complete description of these cohomology groups for lattices in $\mathrm{PU}(r, 1)$.

We proceed as follows: In $\$ 1$ we establish notation, and define and prove some basic facts about the spaces $Q^{*}(\underline{\mu})$. In $\$ 2$ we describe more carefully the relations between these spaces and the work of [DM] and [M], and give their conditions on $\underline{\mu}$ for $\Gamma(\underline{\mu})$ to be a discrete subgroup of $\mathrm{PU}(r, 1)$. In $\S 3$ we divide the sequences of weights into equivalence classes, with equivalent sequences of weights having the same quotient. In $\S 4$ we identify the quotients, in those cases where we are able to do so. When $r=2$, the quotients are complex surfaces, and these surfaces contain particular configurations of lines. We identify these in \$5. (They are related to the configuration studied by Hirzebruch in $[\mathbf{H}]$.)

In $\S 6$ we begin our computation of the cohomology of $Q^{*}(\underline{\mu})$ in those cases not dealt with in $\S 4$. (Indeed, some of the descriptions of $Q^{*}(\underline{\mu})$ in $\S 4$ were first suggested by the computation of the cohomology of these spaces.) Section 6 explains the connection between the spaces we study and Mumford's geometric invariant theory (a connection adumbrated in $\S 1$ ), and $\$ 7$ explains the method of $\left[\mathbf{K}_{\mathbf{1}}\right]$ and $\left[\mathbf{K}_{\mathbf{2}}\right]$ for calculating $H^{*}\left(Q^{*}(\underline{\mu})\right)$, and performs these calculations. Section 8 explains how to calculate the intersection homology of the singular variety $Q(\underline{\mu})$, though here we leave the actual calculations to the reader.

In $\$ 9$ we discuss the question of the representability of homology classes of $Q^{*}(\underline{\mu})$ by algebraic subvarieties.

Finally, the subspace $Q_{\text {st }}(\underline{\mu})$ of $Q^{*}(\underline{\mu})$ is the actual quotient of the ball by the group $\Gamma(\underline{\mu})$. Every point of the ball has finite stabilizer, so the rational cohomology of $Q_{\mathrm{st}}(\underline{\mu})$ is that of $\Gamma(\underline{\mu})$. In the cases we are able to, we compute that cohomology in $\S 10$.

1. Preliminaries. We shall use the language and notation of [DM] here, with some minor differences. Our results here are valid over any 
algebraically closed field $k$. To begin with, we have the following data:

(1.1) $N \geq 3$ an integer, $S=\{1, \ldots, N\}$

$\mu=\left(\mu_{s} \mid s \in S\right)$ a non-increasing sequence of positive integers (i.e. $\mu_{i} \geq \mu_{j}$ if $i<j$ ) with $\Sigma_{s \in S} \mu_{s}=d$ such that $\mu_{s}<d / 2$ for all $s \in S$.

We call a sequence $\mu=\left(\mu_{s}\right)$ as in 1.1 a sequence of weights.

We let $\mathbf{P}=P^{1}(k)$ and $\mathbf{P}^{S}=\left\{\left(y_{s}\right)_{s \in S}\right\}$ be the space of functions from $S$ to $\mathbf{P}$. (We may naturally identify $\mathbf{P}^{S}$ with $\mathbf{P}^{1} \times \cdots \times \mathbf{P}^{1}$, where there are $N$ factors in the product.) We let $M \subset \mathbf{P}^{S}$ be the subspace of injective maps from $S$ to $\mathbf{P}$. The group $\mathrm{PGL}_{2}=\mathrm{PGL}_{2}(k)$ acts on $\mathbf{P} S$ by $g\left(\left(y_{s}\right)_{s \in S}\right)=\left(\left(g y_{s}\right)_{s \in S}\right)$, where $g \in \mathrm{PGL}_{2}$ acts on $\mathbf{P}$ by Möbius transformations. This action leaves $M$ invariant, and we are interested in studying the quotient $Q=\mathrm{PGL}_{2} \backslash M$. More precisely, we are interested in compactifying this quotient.

Definition 1.2. A point $y \in \mathbf{P}^{S}$ is called stable (resp. semi-stable) if for all $z \in \mathbf{P}, \sum_{y(s)=z} \mu_{s}<d / 2$ (resp. $\leq d / 2$ ).

The set of all stable (resp. semi-stable) points is denoted $M_{\text {st }}$ (resp. $M_{\text {sst }}$ ). We have $M \subset M_{\text {st }} \subset M_{\text {sst }}$ and we set $M_{\text {cusp }}=M_{\text {sst }}-M_{\text {st }}$. We call a point $y \in M_{\text {cusp }}$ strictly semi-stable.

If $S_{1}$ is a subset of $S$, we will let $\underline{\mu}\left(S_{1}\right)$ denote $\Sigma_{s \in S_{1}} \mu_{s}$.

For each partition $\left\{S_{1}, S_{2}\right\}$ of $S$ with $\mu\left(S_{i}\right)=d / 2(i=1,2)$, the points $y$ in $\mathbf{P}^{S}$ with $y\left(S_{1}\right) \cap y\left(S_{2}\right)=\varnothing$ and $y$ constant on $S_{1}$ or $S_{2}$ are strictly semi-stable, and each strictly semi-stable point arises in this way, from a unique partition. While the partition only depends on the unordered pair $\left\{S_{1}, S_{2}\right\}$, we adopt the following convention:

(1.3) If $\left\{S_{1}, S_{2}\right\}$ is a partition determining a point in $M_{\text {cusp }}$, then $c\left(S_{1}\right) \leq c\left(S_{2}\right)$, where $c()$ denotes cardinality.

On $M_{\text {sst }}$ we define a relation $\sim$ by $y \sim y^{\prime}$ if and only if

(1.4) (i) $y, y^{\prime} \in M_{\text {st }}$ and are in the same orbit of the action of $\mathrm{PGL}_{2}$,

(ii) $y, y^{\prime} \in M_{\text {cusp }}$ and the partitions determining $y$ and $y^{\prime}$ coincide.

We set

$$
Q_{\text {sst }}=M_{\text {st }} / \sim, \quad Q_{\text {st }}=M_{\text {st }} / \sim, \quad Q_{\text {cusp }}=M_{\text {cusp }} / \sim
$$

each with its quotient topology. Note $Q \subset Q_{\text {st }} \subset Q_{\text {sst }}$, and in fact $Q$ is a Zariski open set in $Q_{\text {sst }}$. The elements of $Q_{\text {cusp }}$ are uniquely determined by their partitions, and so $Q_{\text {cusp }}$ is a finite set (which may be empty). We shall call $q \in Q_{\text {cusp }}$ a cusp. We have:

(1.6) $Q_{\text {sst }}$ is a projective variety, with $\operatorname{dim}_{k} Q_{\text {sst }}=r=N-3$. 
(1.7) $Q_{\text {sst }}$ is non-singular if and only if either $Q_{\text {cusp }}=\varnothing$, or else for all $q \in Q_{\text {cusp }}$, if $\left\{S_{1}, S_{2}\right\}$ is the partition determining $q$, then $c\left(S_{1}\right)=2$. The only singular points of $Q_{\text {sst }}$ are the cusps not satisfying this condition.

The above is all proven in [DM], following [MF]. We shall refine 1.7 in 1.10 below.

Definition 1.8. Let $\underline{\mu}=\left(\mu_{s}\right)_{s \in S}$ and $\underline{\mu}^{\prime}=\left(\mu_{s}^{\prime}\right)_{s \in S}$ be two sequences of weights. We call $\underline{\mu}$ and $\underline{\mu}^{\prime}$ equivalent if they have the following property. For all subsets $\overline{S_{1}}$ of $S, \mu\left(S_{1}\right)<d / 2($ resp. $=d / 2,>d / 2)$ if and only if $\mu^{\prime}\left(S_{1}\right)<d^{\prime} / 2$ (resp. $\left.=d^{\prime} / 2,>d^{\prime} / 2\right)$.

The following is then immediate:

(1.9) If $\underline{\mu}$ and $\underline{\mu}^{\prime}$ are equivalent sequences of weights, then $Q_{\text {sst }}$ defined with respect to $\mu^{\prime}$ may be identified with $Q_{\text {sst }}$ defined with respect to $\mu$, with identification restricting to the identification of cusps in each space determined by the same partition.

Proposition 1.10. Let $Q_{\text {sst }}^{*}$ be the non-singular variety obtained from

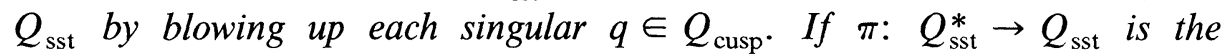
blowdown map, and $q$ is determined by a partition $\left\{S_{1}, S_{2}\right\}$, then $\pi^{-1}(q)=$ $\mathbf{P}^{c\left(S_{1}\right)-2}(k) \times \mathbf{P}^{c\left(S_{2}\right)-2}(k)$.

Proof. For ease of notation, let us assume that $S_{1}=\{1, \ldots, n\}$, $S_{2}=\{n+1, \ldots, N\}$. Let $m \in M_{\text {cusp }}$ be the point $(0,0, \ldots, 0, \infty, \ldots, \infty)$ ( $n$ zeroes and $N-n$ infinities) which projects to $q$. In order to blow up $q$, we must replace it by the set of lines in $M_{\text {st }}$ which pass through $M$, modulo the action of $\mathrm{PGL}_{2}$. Thus

$$
\pi^{-1}(q)=\mathrm{PGL}_{2} \backslash\left\{m^{\prime}=\left(z_{1}, \ldots, z_{n}, w_{n+1}, \ldots, w_{N}\right) \mid m^{\prime} \in M_{\mathrm{st}}\right\} / k^{*}
$$

where the action of $k^{*}$ on the right is the usual action

$$
\left(z_{1}, \ldots, z_{n}, w_{n+1}, \ldots, w_{N}\right) t=\left(z_{1} t, \ldots, z_{n} t, w_{n+t}, \ldots, w_{N} t\right)
$$

which identifies points which are on the same line.

The condition $m^{\prime} \in M_{\text {st }}$ means that not all the $z_{l}$ can be zero, and not all the $w_{j}$ can be $\infty$. Since we are interested in a neighborhood of $m$ we can assume that the $z_{i}$ are near zero and the $w_{i}$ are near $\infty$, so that in particular $z_{i} \neq w_{j}$.

In order to analyze the quotient we may, after acting on $m^{\prime}$ by a suitable element of $\mathrm{PGL}_{2}$, assume that $z_{1}=0$ and $w_{n+1}=\infty$. Then we must further divide out by the subgroup of $\mathrm{PGL}_{2}$ which fixes 0 and $\infty$, which we may identify with $k^{*}$. Thus

$$
\pi^{-1}(q)=k^{*} \backslash\left\{\left(0, z_{2}, \ldots, z_{n}, \infty, w_{n+2}, \ldots, w_{N}\right)\right\} / k^{*} .
$$


Note, however, that the action of $k^{*}$ on the left is given by

$$
\begin{aligned}
& \lambda\left(0, z_{2}, \ldots, z_{n}, \infty, w_{n+2}, \ldots, w_{N}\right) \\
& \quad=\left(0, \lambda z_{2}, \ldots, \lambda z_{n}, \infty, \lambda^{-1} w_{n+2}, \ldots, \lambda^{-1} w_{N}\right) .
\end{aligned}
$$

Since for any $u, v \in k^{*}$ we may simultaneously solve $\lambda t=u, \lambda^{-1} t=v$, it readily follows that $\pi^{-1}(q)=\mathbf{P}^{n-2}(k) \times \mathbf{P}^{N-n-2}(k)$, as claimed.

Proposition 1.11. For any sequence of weights $\mu$ satisfying conditions (1.1), $Q^{*}(\underline{\mu})$ is rational.

Proof. $Q$ is a Zariski open set in $Q^{*}(\underline{\mu})$. Also,

$$
\begin{aligned}
Q=\mathrm{PGL}_{2} \backslash M=\mathrm{PGL}_{2} \backslash\left\{\left(z_{1}, \ldots, z_{n}\right\} \mid z_{i} \neq z_{j}\right\} \\
=\left\{\left(\infty, 0,1, z_{4}, \ldots, z_{N}\right) \mid z_{i} \neq z_{j}, z_{i} \neq 0,1, \infty\right\}
\end{aligned}
$$

which, under the projection onto the last $N-3$ coordinates, may be identified with a Zariski open set in $\left(\mathbf{P}^{1}\right)^{N-3}$. Hence $Q^{*}(\underline{\mu})$ is birationally equivalent to $\left(\mathbf{P}^{1}\right)^{N-3}$, and so is rational.

2. The work of Deligne and Mostow. In this section we briefly recall the appearance of the varieties $Q_{\text {sst }}$ in the work of [DM] and [M]. They showed that in the case $k=\mathbf{C}$ they arise as follows:

Let $\lambda_{i}=2 \mu_{i} / d$, and consider the path integrals, for $i \geq 2$ and $\left(z_{1}, \cdots, z_{\mathrm{N}}\right) \in M$

$$
\begin{aligned}
F_{i}\left(z_{1}, \ldots, z_{N}\right) & =\int_{z_{i}}^{z_{1}} \prod_{i=2}^{N}\left(u-z_{i}\right)^{-\lambda_{i}} d u \quad \text { when } z_{1}=\infty \\
& =\int_{z_{i}}^{z_{1}} \prod_{i=1}^{N}\left(u-z_{i}\right)^{-\lambda_{i}} d u \quad \text { when all } z_{i} \text { are finite. }
\end{aligned}
$$

(Note we may pass from the first of these to the second by a Möbius transformation.) It turns out that there are $(r+1)$ linearly independent integrals among these. Of course, these integrals are not yet well-defined, as they depend on the choice of a path of integration. Thus by choosing such an independent set we obtain a multi-valued map from $M$ to $\mathrm{C}^{r+1}-\{0\}$. The action of $\mathrm{PGL}_{2}$ on $M$ multiplies each of these integrals by the same factor, so we obtain a multi-valued map from $Q=\mathrm{PGL}_{2} \backslash M$ to $\mathbf{P}^{r}$, or, more precisely, a well-defined map from the universal cover $\tilde{Q}, \tilde{f}: \tilde{Q} \rightarrow \mathbf{P}^{r}$. This map $\tilde{f}$ is equivariant under the action of $\pi_{1}(Q)$ by covering translations, and so gives a map $\pi_{1}(Q) \rightarrow \operatorname{Aut}\left(\mathbf{P}^{N-3}\right)=$ PGL $(N-2)$ with image $\Gamma$. If $\mu$ satisfies condition INT below then $\Gamma$ is discrete in $\operatorname{PU}(N-3,1) \subset \operatorname{PGL}(N-2)$, in which case $\Gamma$ has a fundamental domain for its action on a ball $B^{+}$in $\mathbf{P}^{N-3}$ and $Q_{\mathrm{st}}=B^{+} / \Gamma$. In 
fact, the action of $\Gamma$ extends to the closure $\bar{B}^{+}$of $B^{+}$(in an unusual topology) and $Q_{\text {sst }}=\bar{B}^{+} / \Gamma$.

In case $\underline{\mu}$ satisfies a weaker condition $\Sigma$ INT below then a variation of the construction applies: Let $Q^{\prime}$ be the subset of $Q$ consisting of the image of points $y \in \mathbf{P}^{S}$ with $y(i) \neq y(j)$ for $i, j \in S_{1}$, so that the symmetric group $\Sigma=\Sigma_{n}, n=c\left(S_{1}\right)$, operates freely on $Q^{\prime}$. The quotient $Q^{\prime} / \Sigma$ plays the role of $Q$ above, giving a discrete subgroup $\Gamma_{\Sigma}$ of $\mathrm{PU}(N-3,1)$. (In case $\underline{\mu}$ satisfies INT as well this construction gives a diagram

$$
\begin{aligned}
& Q_{\text {sst }}=\bar{B}^{+} / \Gamma \\
& \downarrow \\
& Q_{\text {sst }} / \Sigma=\bar{B}^{+} / \Gamma_{\Sigma}
\end{aligned}
$$

with $\Gamma_{\Sigma} / \Gamma=\Sigma_{n}$.) However, in this case $Q_{\text {st }}$ will only be a $V$-manifold, i.e. will have finite quotient singularities.

(2.1) INT: For all $i \neq j \in S$ such that $\mu_{\imath}+\mu_{j}<d / 2,1-$ $2\left(\mu_{i}+\mu_{j}\right) / d$ is the reciprocal of an integer.

(2.2) $\Sigma$ INT: There is a subset $S_{1}$ of $S$ with $\mu_{\imath}=\mu_{j}$ for all $i \neq j \in S_{1}$ with $\mu_{i}+\mu_{j}<d / 2$, with $1-2\left(\mu_{i}+\mu_{j}\right) / d$ the reciprocal of an integer if either $i$ or $j$ is not in $S_{1}$, and $1-2\left(\mu_{i}+\mu_{j}\right) / d$ the reciprocal of a half-integer if both $i$ and $j$ are in $S_{1}$.

(In Tables I and II below the size of the symmetric group $\Gamma$ is obvious from the entries.)

Deligne and Mostow also derive the following condition for $\Gamma$ to be arithmetic in $\mathrm{PU}(N-3,1)$.

(2.3) ARITH: Assume the highest common factor of $\left(\mu_{s}\right)_{s \in S}$ is one. (Otherwise, first divide the $\mu_{s}$ by this factor.) Let $\langle x\rangle$ denote the fractional part of $x$, i.e. $\langle x\rangle=x-[x]$.

If $d$ is even, then for all $A$ with $1<A<d / 2-1,(A, d / 2)=1$,

$$
\sum_{s \in S}\left\langle 2 A \mu_{s} / d\right\rangle=1 \text { or } N-1 \text {. }
$$

If $d$ is odd, then for all $A$ with $1<A<d / 2,(A, d)=1$,

$$
\sum_{s \in S}\left\langle 2 A \mu_{s} / d\right\rangle=1 \text { or } N-1 \text {. }
$$

3. Classification of the weights. Now we begin the analysis of the varieties $Q_{\text {sst }}$. The first step in the analysis is to divide the sequences of weights into equivalence classes. From 1.8, this is a routine (but lengthy) computation, and the results are to be found in Table I (for $N=5$ ) and Table II (for $N>5$ ). In both tables $N$ denotes the number of weights, $\underline{\mu}$ 
TABLE I

\begin{tabular}{|c|c|c|c|c|c|c|c|c|c|}
\hline \multirow{2}{*}{$\frac{N . m}{5.1}$} & \multicolumn{5}{|c|}{$\underline{\mu}$} & \multirow{2}{*}{$\frac{\infty_{n}}{-}$} & \multirow{2}{*}{$\frac{\infty_{s}}{-}$} & \multirow[t]{2}{*}{$\Sigma$} & \multirow[t]{2}{*}{$\mathrm{A} / \mathrm{NA}$} \\
\hline & 1 & 1 & 1 & 1 & 1 & & & & \\
\hline & 2 & 2 & 2 & 2 & 1 & & & & \\
\hline & 4 & 3 & 3 & 3 & 2 & & & & NA \\
\hline & 4 & 3 & 3 & 3 & 3 & & & & \\
\hline & 5 & 5 & 5 & 5 & 4 & & & & \\
\hline & 6 & 5 & 5 & 4 & 4 & & & & NA \\
\hline & 6 & 5 & 5 & 5 & 3 & & & & NA \\
\hline & 8 & 5 & 5 & 5 & 5 & & & $\Sigma$ & \\
\hline & 8 & 7 & 7 & 7 & 7 & & & $\Sigma$ & NA \\
\hline & 10 & 7 & 7 & 7 & 5 & & & $\Sigma$ & NA \\
\hline & 10 & 9 & 9 & 6 & 6 & & & $\Sigma$ & NA \\
\hline & 14 & 9 & 9 & 9 & 7 & & & & NA \\
\hline \multirow[t]{11}{*}{5.2} & 5 & 5 & 2 & 2 & 2 & - & - & & \\
\hline & 6 & 5 & 3 & 3 & 3 & & & $\Sigma$ & \\
\hline & 7 & 7 & 4 & 4 & 2 & & & & \\
\hline & 8 & 7 & 3 & 3 & 3 & & & & NA \\
\hline & 9 & 9 & 2 & 2 & 2 & & & $\Sigma$ & \\
\hline & 13 & 9 & 6 & 6 & 6 & & & $\Sigma$ & NA \\
\hline & 14 & 11 & 5 & 5 & 5 & & & & NA \\
\hline & 14 & 13 & 3 & 3 & 3 & & & $\Sigma$ & \\
\hline & 19 & 17 & 4 & 4 & 4 & & & $\Sigma$ & NA \\
\hline & 23 & 22 & 5 & 5 & 5 & & & $\Sigma$ & NA \\
\hline & 34 & 29 & 7 & 7 & 7 & & & $\Sigma$ & NA \\
\hline \multirow[t]{4}{*}{5.3} & 6 & 3 & 3 & 3 & 1 & - & - & & \\
\hline & 7 & 4 & 4 & 4 & 1 & & & & \\
\hline & 8 & 5 & 5 & 5 & 1 & & & & \\
\hline & 11 & 8 & 8 & 8 & 1 & & & & \\
\hline \multirow[t]{6}{*}{5.4} & 8 & 3 & 3 & 3 & 3 & - & - & $\Sigma$ & \\
\hline & 9 & 3 & 3 & 3 & 2 & & & $\Sigma$ & \\
\hline & 10 & 5 & 3 & 3 & 3 & & & & \\
\hline & 11 & 7 & 2 & 2 & 2 & & & $\Sigma$ & \\
\hline & 22 & 11 & 9 & 9 & 9 & & & $\Sigma$ & \\
\hline & 26 & 19 & 5 & 5 & 5 & & & $\Sigma$ & \\
\hline 5.5 & 8 & 5 & 5 & 3 & 3 & - & - & & \\
\hline 5.6 & 2 & 1 & 1 & 1 & 1 & 4 & - & & \\
\hline \multirow[t]{2}{*}{5.7} & 2 & 2 & 2 & 1 & 1 & 3 & - & & \\
\hline & 3 & 3 & 3 & 2 & 1 & & & & \\
\hline \multirow[t]{2}{*}{5.8} & 3 & 2 & 1 & 1 & 1 & 3 & - & & \\
\hline & 5 & 4 & 1 & 1 & 1 & & & $\Sigma$ & \\
\hline \multirow[t]{2}{*}{5.9} & 3 & 3 & 2 & 2 & 2 & 1 & - & & \\
\hline & 7 & 5 & 4 & 4 & 4 & & & & NA \\
\hline 5.10 & 4 & 3 & 2 & 2 & 1 & 2 & - & & \\
\hline 5.11 & 5 & 2 & 2 & 2 & 1 & 1 & - & & \\
\hline 5.12 & 5 & 3 & 2 & 1 & 1 & 2 & - & $\Sigma$ & \\
\hline 5.13 & 7 & 6 & 5 & 3 & 3 & 1 & - & & NA \\
\hline
\end{tabular}


TABLE II

\begin{tabular}{lllllllllll}
\hline$N$ & & & $\underline{\mu}$ & & & $\infty_{n}$ & $\infty_{s}$ & $\Sigma$ A/NA \\
\hline 6 & 3 & 3 & 3 & 3 & 3 & 1 & - & - & & \\
\hline 6 & 5 & 3 & 3 & 3 & 3 & 3 & - & - & $\Sigma$ \\
& 6 & 3 & 3 & 3 & 3 & 2 & & & $\Sigma$ \\
\hline 6 & 5 & 5 & 5 & 3 & 3 & 3 & - & - & & \\
\hline 6 & 3 & 1 & 1 & 1 & 1 & 1 & 5 & - & & \\
\hline 6 & 3 & 3 & 3 & 1 & 1 & 1 & 3 & - & $\Sigma$ \\
\hline 6 & 5 & 2 & 2 & 1 & 1 & 1 & 3 & - & $\Sigma$ \\
\hline 6 & 5 & 3 & 1 & 1 & 1 & 1 & 4 & - & $\Sigma$ \\
\hline 6 & 7 & 5 & 3 & 3 & 3 & 3 & 1 & - & & NA \\
\hline 6 & 1 & 1 & 1 & 1 & 1 & 1 & - & 10 & \\
\hline 6 & 2 & 2 & 1 & 1 & 1 & 1 & 1 & 6 & \\
\hline 6 & 3 & 2 & 2 & 2 & 2 & 1 & - & 4 & \\
\hline 6 & 4 & 3 & 2 & 1 & 1 & 1 & 1 & 3 & $\Sigma$ \\
\hline 6 & 4 & 4 & 1 & 1 & 1 & 1 & - & 6 & $\Sigma$ \\
\hline 7 & $3^{6}$ & 2 & & & & & - & $\Sigma$ \\
\hline 7 & 7 & 7 & $2^{5}$ & & & & - & $\Sigma$ \\
\hline 7 & 5 & 2 & $1^{5}$ & & & 5 & - & $\Sigma$ \\
\hline 7 & 2 & $1^{6}$ & & & & - & 15 & \\
\hline 7 & 3 & 3 & 2 & $1^{4}$ & & 1 & 8 & $\Sigma$ \\
\hline 7 & 4 & 2 & 2 & $1^{4}$ & & 2 & 6 & $\Sigma$ \\
\hline 7 & 4 & 3 & $1^{5}$ & & & - & 10 & $\Sigma$ \\
\hline 8 & 5 & $1^{7}$ & & & & 7 & - & $\Sigma$ \\
\hline 8 & $1^{8}$ & & & & & - & 35 & $\Sigma$ \\
\hline 8 & 3 & 2 & 2 & $1^{5}$ & & - & 30 & $\Sigma$ \\
\hline 8 & 3 & 3 & $1^{6}$ & & & 1 & 20 & $\Sigma$ \\
\hline 8 & 4 & 2 & $1^{6}$ & & & 1 & 15 & $\Sigma$ \\
\hline 9 & 2 & 2 & 2 & $1^{6}$ & & - & 46 & $\Sigma$ \\
\hline 9 & 3 & 2 & $1^{7}$ & & & - & 42 & $\Sigma$ \\
\hline 9 & 4 & $1^{8}$ & & & & - & 28 & $\Sigma$ \\
\hline 10 & 2 & 2 & $1^{8}$ & & & - & 98 & $\Sigma$ \\
\hline 10 & 3 & $1^{9}$ & & & & - & 84 & $\Sigma$ \\
\hline 11 & 2 & $1^{10}$ & & & & - & 210 & $\Sigma$ \\
\hline 12 & $1^{12}$ & & & & & & 462 & $\Sigma$ \\
\hline & & & & & & & &
\end{tabular}


the sequence of weights, $\infty_{n}$ the number of non-singular cusps and $\infty_{s}$ the number of singular cusps (so that $Q_{\text {st }}$ is compact if $\infty_{n}+\infty_{s}=0$, and $Q_{\text {sst }}$ is non-singular if $\infty_{s}=0$ ). Also, the column headed $\Sigma$ is blank if $\underline{\mu}$ satisfies INT, but is labelled $\Sigma$ if it satisfies $\Sigma$ INT but not INT, and the column A/NA is blank if $\mu$ satisfies ARITH, but is labelled NA if not.

Because of the many elements of several of the equivalence classes in Table I, we have numbered the equivalence classes 5.1-5.13 and will refer to a sequence of weights being of type $5 . \mathrm{m}$. In Table II we have used $\mathrm{m}^{n}$ to denote a sequence of $n$ values of $m$.

Finally, these tables are arranged so that for a given $N$, the compact quotients are first, followed by the non-singular quotients. Subject to this restriction, the equivalence classes are listed in lexicographic order.

4. The structure of $Q(\underline{\mu})$. We let $Q(\underline{\mu})$ be the variety previously denoted by $Q_{\text {sst }}$, where the criteria for stability and semi-stability are with respect to the sequence of weights $\mu$. We let $Q^{*}(\underline{\mu})$ be the non-singular variety obtained by blowing up the singular cusps of $Q(\mu)$.

We shall now completely analyze the structure of $\bar{Q}(\mu)$ in those cases where it is non-singular, with the exception of the cases $N=7, \underline{\mu}=3^{6} 2$ or $772^{5}$. We remind the reader that our results here are valid over an arbitrary algebraically closed field (of any characteristic).

THEOREM 4.1.

(i) If $\underline{\mu}$ is of type 5.1, $Q(\underline{\mu})$ is $\mathbf{P}^{2}$ with 4 points blown up.

(ii) If $\underline{\mu}$ is of type 5.2 or $5.9, Q(\underline{\mu})$ is $\mathbf{P}^{2}$ with 3 points blown up.

(iii) If $\underline{\mu}$ is of type 5.5 or $5.13, \overline{Q(\mu)}$ is $\mathbf{P}^{2}$ with 2 points blown up.

(iv) If $\underline{\mu}$ is of type $5.7, Q(\underline{\mu})$ is $\mathbf{P}^{\overline{1}} \times \mathbf{P}^{1}$.

(v) If $\underline{\mu}$ is of type 5.3 or $5.10, Q(\underline{\mu})$ is $\mathbf{P}^{2}$ with 1 point blown up.

(vi) If $\underline{\mu}$ is of type $5.4,5.6,5.8,5 . \overline{1}$, or $5.12, Q(\underline{\mu})$ is $\mathbf{P}^{2}$.

(vii) $Q(\bar{l} 3333331)$ is a $\mathbf{P}^{1}$ bundle over $\left(\mathbf{P}^{2}\right.$ with $\overline{4}$ points blown up).

(viii) $Q\left(\begin{array}{lllll}5 & 3 & 3 & 3 & 3\end{array}\right)=Q\left(\begin{array}{lllll}6 & 3 & 3 & 3 & 3\end{array}\right)$ is a $\left(\mathbf{P}^{1}\right.$ bundle over $\left.\mathbf{P}^{2}\right)$ with 4 points blown up. This space can also be described as $\mathbf{P}^{3}$ with 5 points blown up.

(ix) $Q\left(\begin{array}{l}5 \\ 5\end{array} 5333\right.$ ) is $\mathbf{P}^{1} \times \mathbf{P}^{1} \times \mathbf{P}^{1}$ with 3 points blown up.

(x) $Q$ (3 331111 ) is $\mathbf{P}^{1} \times \mathbf{P}^{1} \times \mathbf{P}^{1}$.

(xi) $Q(753333)$ is $Q(533333)$ with one $\mathbf{P}^{2}$ blown down.

(xii) $Q(3111111)$ is $\mathbf{P}^{3}$.

(xiii) $Q(522111)$ is $\mathbf{P}^{3}$.

(xiv) $Q(531111)$ is $\mathbf{P}^{3}$.

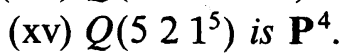

(xvi) $Q\left(51^{7}\right)$ is $\mathbf{P}^{5}$. 
Proof. We shall only prove cases (vii)-(xi) here. Cases (i)-(vi), with $N=5$, are less interesting, and (xii) $-(\mathrm{xvi})$ are special cases of 4.2 below. We denote a point in $\left(\mathbf{P}^{1}\right)^{6}$ by $\left(z_{1}, \ldots, z_{6}\right)$.

(vii): Let $\pi:\left(\mathbf{P}^{1}\right)^{6} \rightarrow\left(\mathbf{P}^{1}\right)^{5}$ be projection on the first 5 coordinates. It is easy to check that $\pi$ descends to a map

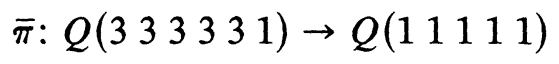

which is a $\mathbf{P}^{1}$ bundle (as $z_{6}$ may be chosen arbitrarily without destroying stability), and $Q\left(\begin{array}{lllll}1 & 1 & 1 & 1 & 1\end{array}\right)$ is $\mathbf{P}^{2}$ with 4 points blown up by part (i).

(viii): Again consider the map $\bar{\pi}: Q\left(\begin{array}{llllll}6 & 3 & 3 & 3 & 3 & 2\end{array}\right) \rightarrow Q\left(\begin{array}{lllll}6 & 3 & 3 & 3 & 3\end{array}\right)$ $=Q\left(\begin{array}{lllll}2 & 1 & 1 & 1 & 1\end{array}\right)$ induced by $\pi$. Again it is easy to check that the inverse image of a stable point in $Q\left(\begin{array}{lllll}2 & 1 & 1 & 1 & 1\end{array}\right)$ is $\mathbf{P}^{1}$ (as $z_{6}$ may be chosen arbitrarily). There are four cusps. The situation at each is the same, so it suffices to check that cusp $q$ determined by the partition $\{\{1,2\},\{3,4,5\}\}$

$$
\bar{\pi}^{-1}(q)=\left\{\left(z_{1}, \ldots, z_{6}\right) \mid \text { either } z_{1}=z_{2} \text { or } z_{3}=z_{4}=z_{5}\right\} \text {. }
$$

Let $V_{1}$ be the subset of $\bar{\pi}^{-1}(q)$ consisting of points with $z_{1}=z_{2}$, and $V_{2}$ be the subset consisting of points with $z_{3}=z_{4}=z_{5}$. First note that

$$
V_{1} \cap V_{2}=\mathrm{PGL}_{2} \backslash\left\{(z, z, w, w, w, u) \in\left(\mathbf{P}^{1}\right)^{6} \mid z, w, u \text { distinct }\right\} .
$$

(The restriction on $z, w$, and $u$ is due to the requirement of semi-stability.) Since under the action of $\mathbf{P G L}_{2}$, any 3 distinct points in $\mathbf{P}^{1}$ are equivalent to any other 3 points, $V_{1} \cap V_{2}=\{(0,0,1,1,1, \infty)\}$ is a single point.

Now consider $V_{2}=\mathrm{PGL}_{2} \backslash\left\{\left(z_{1}, z_{2}, w, w, w, z_{6}\right) \mid z_{i} \neq w, z_{1}, z_{2}, z_{6}\right.$ not all equal $\}$. (Again, the restrictions are due to the requirement of semistability. Henceforth, we will state this sort of restriction without comment.)

We may use the action of $\mathrm{PGL}_{2}$ to send $z_{1}$ to 0 and $w$ to $\infty$, whence we must take the quotient by the subgroup of $\mathrm{PGL}_{2}$ fixing 0 and $\infty$, which we shall identify with $k^{*}$. Thus

$$
V_{2}=k^{*} \backslash\left\{\left(0, z_{1}, \infty, \infty, \infty, z_{6}\right) \mid z_{2} \neq \infty, z_{6} \neq \infty,\left(z_{2}, z_{6}\right) \neq(0,0)\right\},
$$

and so $V_{2}=\mathbf{P}^{1}$.

As for $V_{1}=\mathrm{PGL}_{2} \backslash\left\{\left(w, w, z_{3}, z_{4}, z_{5}, z_{6}\right) \mid z_{i} \neq w\right.$, and $z_{3}, z_{4}, z_{5}, z_{6}$ not all equal $\}$, we may use the action of $\mathrm{PGL}_{2}$ to send $w$ to $\infty$ and $z_{3}$ to 0 . Thus

$$
\begin{aligned}
V_{1}=k^{*} \backslash\left\{\left(\infty, \infty, 0, z_{4}, z_{5}, z_{6}\right) \mid z_{4}\right. & \neq \infty, z_{5} \neq \infty, \\
z_{6} & \left.\neq \infty,\left(z_{4}, z_{5}, z_{6}\right) \neq(0,0,0)\right\},
\end{aligned}
$$

and so $V_{1}=\mathbf{P}^{2}$. 
Hence $\bar{\pi}^{-1}(q)$ is the one-point union of $\mathbf{P}^{1}$ and $\mathbf{P}^{2}$. One can check that the $\mathbf{P}^{2}$ can be blown down to a point, giving $\pi^{-1}(q)=\mathbf{P}^{1}$, and that in the neighborhood of $q$ one still has a bundle structure, so $Q\left(\begin{array}{lllll}6 & 3 & 3 & 3 & 2\end{array}\right)$ $=Q\left(\begin{array}{lllll}5 & 3 & 3 & 3 & 3\end{array}\right)$ is as claimed.

For the second description, consider the subset $U \subset Q\left(\begin{array}{lllllll}6 & 3 & 3 & 3 & 3 & 2\end{array}\right)$ of points with $z_{i} \neq z_{1}$ for $i>1$. Then

$$
\begin{aligned}
U & =\mathrm{PGL}_{2} \backslash\left\{\left(z_{1}, \ldots, z_{6}\right) \mid z_{i} \neq z_{1} \text { for } i>1, \text { at most } 3 \text { of } z_{2}, \ldots, z_{6} \text { equal }\right\} \\
& =k^{*} \backslash\left\{\left(\infty, 0, z_{3}, \ldots, z_{6}\right) \mid \text { at most } 2 \text { of } z_{3}, \ldots, z_{6} \text { equal to } 0,\right.
\end{aligned}
$$

at most 3 of $z_{3}, \ldots, z_{6}$ equal $\}$

$$
=\mathbf{P}^{3}-([0,0,0,1] \cup[0,0,1,0] \cup[0,1,0,0] \cup[1,0,0,0] \cup[1,1,1,1]) \text {. }
$$

The space $Q\left(\begin{array}{llllll}6 & 3 & 3 & 3 & 3\end{array}\right)$ is covered by $U$ and sets $V_{1 j}, j=2, \ldots, 6$, where $V_{1 j}$ denotes the set of points where $z_{1}=z_{j}$. Clearly all the $V_{1 j}$ have the same structure, so it suffices to consider $V_{12}$ :

$$
\begin{aligned}
V_{12}= & \mathrm{PGL}_{2} \backslash\left\{\left(w, w, z_{3}, z_{4}, z_{5}, z_{6}\right) \mid z_{i} \neq w,\right. \\
& \left.\quad \text { and } z_{3}, z_{4}, z_{5}, z_{6} \text { not all equal }\right\} \\
= & k^{*} \backslash\left\{\left(\infty, \infty, 0, z_{4}, z_{5}, z_{6}\right) \mid z_{i} \neq \infty,\left(z_{4}, z_{5}, z_{6}\right) \neq(0,0,0)\right\}=\mathbf{P}^{2} .
\end{aligned}
$$

One can check that a neighborhood of $V_{1 j}$ has the structure of a blow-up, so $Q\left(\begin{array}{llllll}6 & 3 & 3 & 3 & 3 & 2\end{array}\right)$ is as claimed.

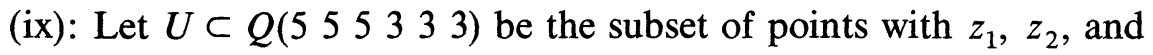
$z_{3}$ distinct. Then

$$
\begin{aligned}
U= & \mathrm{PGL}_{2} \backslash\left\{\left(z_{1}, \ldots, z_{6}\right) \mid z_{1} \neq z_{2} \neq z_{3} \neq z_{1},\right. \\
& \left.\left(z_{4}, z_{5}, z_{6}\right) \neq\left(z_{i}, z_{i}, z_{i}\right) i=1,2, \text { or } 3\right\} \\
= & \left\{\left(0,1, \infty, z_{4}, z_{5}, z_{6}\right) \mid\left(z_{4}, z_{5}, z_{6}\right) \neq(0,0,0),(1,1,1),(\infty, \infty, \infty)\right\} \\
= & \mathbf{P}^{1} \times \mathbf{P}^{1} \times \mathbf{P}^{1}-((0,0,0) \cup(1,1,1) \cup(\infty, \infty, \infty)) .
\end{aligned}
$$

The space $Q\left(\begin{array}{llllll}5 & 5 & 3 & 3 & 3\end{array}\right)$ is covered by $U$ and $V_{12}, V_{13}, V_{23}$, where $V_{i j}$ denotes the set of points where $z_{i}=z_{j}$. Clearly, $V_{12}, V_{13}$, and $V_{23}$ all have the same structure, so it suffices to consider $V_{12}$ :

$$
\begin{aligned}
V_{12}= & \mathrm{PGL}_{2} \backslash\left\{\left(w, w, z_{3}, z_{4}, z_{5}, z_{6}\right) \mid z_{i} \neq w,\right. \\
& \text { and } \left.z_{3}, z_{4}, z_{5}, z_{6} \text { not all equal }\right\} / \mathrm{PGL}_{2} \\
= & k^{*} \backslash\left\{\left(\infty, \infty, 0, z_{4}, z_{5}, z_{6}\right) \mid z_{i} \neq \infty,\left(z_{4}, z_{5}, z_{6}\right) \neq(0,0,0)\right\}=\mathbf{P}^{2} .
\end{aligned}
$$

One can again check that a neighborhood $V_{12}$ has the structure of a blow-up, so $Q\left(\begin{array}{lllll}5 & 5 & 3 & 3 & 3\end{array}\right)$ is as claimed. 


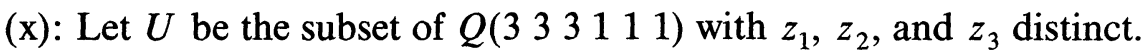
Using the action of $\mathrm{PGL}_{2}$, we may let

$$
U=\left\{\left(0,1, \infty, z_{4}, z_{5}, z_{6}\right) \mid z_{i} \in \mathbf{P}^{1}\right\} / \sim
$$

where $\sim$ is the relation of 1.4 , which we must consider in this case as $Q\left(\begin{array}{llllll}3 & 3 & 3 & 1 & 1 & 1\end{array}\right)$ has cusps.

This relation implies that points with $z_{4}=z_{5}=z_{6}=0$ must be identified to a point (and also $=1$ or $=\infty$ ), but this has no effect as such a condition already defines a single point. Hence we see that

$$
U=\mathbf{P}^{1} \times \mathbf{P}^{1} \times \mathbf{P}^{1} .
$$

Now we must consider points not in $U$. Let $V_{23}$ be the points with $z_{2}=z_{3}$. Again, such points are strictly semi-stable, and so define a cusp determined by the partition $\{\{2,3\},\{1,4,5,6\}\}$, which is the same as the cusp defined by $z_{4}=z_{5}=z_{6}=0$ considered above, and similarly for points with $z_{1}=z_{3}$ or $z_{1}=z_{2}$. Thus $Q\left(\begin{array}{l}3 \\ 3\end{array} 31111\right)$ is as claimed.

(xi): Consider the identity map $i:\left(\mathbf{P}^{1}\right)^{6} \rightarrow\left(\mathbf{P}^{1}\right)^{6}$. The map $i$ descends

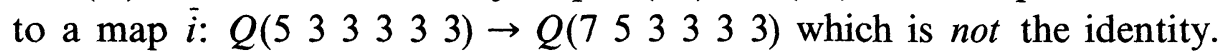
However, it is easy to check that the inverse image of any stable point of

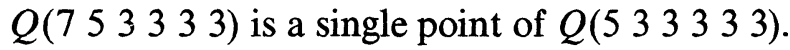

This leaves us to determine $\vec{i}^{-1}(q)$, where $q$ is the (unique) cusp of

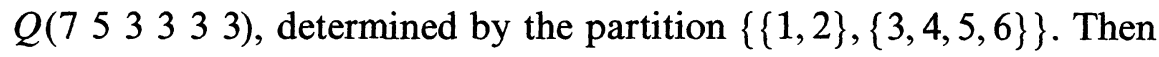

$$
\begin{aligned}
\bar{i}^{-1}(q) & =\mathrm{PGL}_{2} \backslash\left\{\left(w, w, z_{3}, z_{4}, z_{5}, z_{6}\right) \mid z_{i} \neq w, \text { not all } z_{i} \text { equal }\right\} \\
& =k^{*} \backslash\left\{\left(\infty, \infty, 0, z_{4}, z_{5}, z_{6}\right) \mid z_{i} \neq \infty,\left(z_{4}, z_{5}, z_{6}\right) \neq(0,0,0)\right\} \\
& =\mathbf{P}^{2}
\end{aligned}
$$

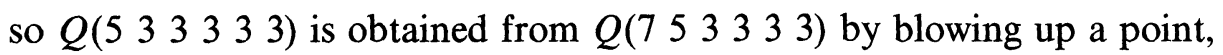
and the result follows.

THEOREM 4.2. Let $\mu$ be a sequence of weights such that $\mu_{1}+\mu_{N} \geq d / 2$ (and hence $\mu_{1}+\mu_{i} \geq d / 2$ for all $\left.i=2, \ldots, N\right)$. Then $Q(\underline{\mu})=\mathbf{P}^{N-3}$.

Proof. Assume first that $\mu_{1}+\mu_{2}>d / 2$. Then $z_{1} \neq z_{2}$, so using the action of $\mathrm{PGL}_{2}$ we may let $z_{1}=\infty, z_{2}=0$, whence

$$
Q(\underline{\mu})=k^{*} \backslash\left\{\left(\infty, 0, z_{3}, \ldots, z_{N}\right)\right\} / \sim \text {. }
$$

If $\mu_{1}+\mu_{N}>d / 2$ then there are no strictly semi-stable points, so the relation $\sim$ of 1.4 (ii) does nothing, and stability requires $z_{i} \neq \infty$, $i=3, \ldots, N$, and $\left(z_{3}, \ldots, z_{N}\right) \neq(0, \ldots, 0)$ so $Q(\underline{\mu})$ is $\mathbf{P}^{N-3}$. 
If $\mu_{1}+\mu_{i}=d / 2$ for $i=N$ (and similarly for other values as well) we have a non-singular cusp determined by the partition $\{\{1, N\},\{2, \ldots$, $N-1\}\}$. While this cusp can be represented by points with $z_{1}=z_{N}$, it can also be represented by points with $z_{2}=\cdots=z_{N-1}$ and distinct from $z_{1}$ and $z_{N}$, which we may take distinct from each other, i.e. by a point $\left\{\left(\infty, 0, \ldots, 0, z_{N}\right)\right\}$. These points have already been considered in the previous paragraph, and they are already equivalent under the action of $k^{*}$ to a single point, so this case changes nothing.

Finally, if $\mu_{1}+\mu_{2}=d / 2$, we have a cusp determined by the partition $\{\{1,2\},\{3, \ldots, N\}\}$, so it has a representative of the form $\{(\infty, 0, z, \ldots, z)\}$, and the argument is as above.

5. Configurations of lines in surfaces. We have determined the structure of all of the surfaces in 4.1. There are natural configurations of projective lines on these surfaces: On the surface $Q(\underline{\mu})$ there are projective lines $\Delta_{i j}=\left\{z_{i}=z_{j}\right\}$ whenever $\mu_{i}+\mu_{j}<d / 2$. (Note that if $Q(\underline{\mu})$ contains $\Delta_{i j}$ and $\Delta_{k l}$ with $i, j, k, l$ distinct, they intersect in a double point, while if $Q(\underline{\mu})$ contains $\Delta_{i j}, \Delta_{j k}$, and $\Delta_{i k}$ with $i, j, k$ distinct, they intersect in a triple point.) In Figure I (i) -(vi) we shall draw these configurations. It is routine to verify that they are correct.

Although each case (i)-(vi) of 4.10 may involve several equivalence classes of sequences of weights, the configurations do not depend on the equivalence classes, but only on the appropriate case of 4.1 . What differs among the equivalence classes is the number and location of the cusps, which are always triple points.

We number the cases in Figure I below to correspond to the cases of 4.1. We denote the line $\Delta_{i j}$ by $i j$. Thus a triple point is denoted $i j k$. The cusp situation is as follows (with the same numbering):

(i) $\underline{\mu}$ of type $5.1-$ no cusps

(ii) $\underline{\mu}$ of type $5.2-$ no cusps

$\underline{\mu}$ of type $5.9-1$ cusp, the triple point 345

(iii) $\underline{\mu}$ of type $5.5-$ no cusps

$\underline{\mu}$ of type $5.13-1$ cusp, the triple point 245

(iv) $\underline{\mu}$ of type $5.7-3$ cusps, the triple points 145,245 , and 345

(v) $\underline{\mu}$ of type $5.3-$ no cusps

$\underline{\mu}$ of type $5.10-2$ cusps, the triple points 245 and 345

(vi) $\underline{\mu}$ of type $5.4-$ no cusps

$\underline{\mu}$ of type $5.6-4$ cusps, all of the triple points

$\underline{\mu}$ of type $5.8-3$ cusps, the triple points $234,235,245$

$\underline{\mu}$ of type $5.11-1$ cusp, the triple point 234

$\underline{\mu}$ of type $5.12-2$ cusps, the triple points 234 and 235 


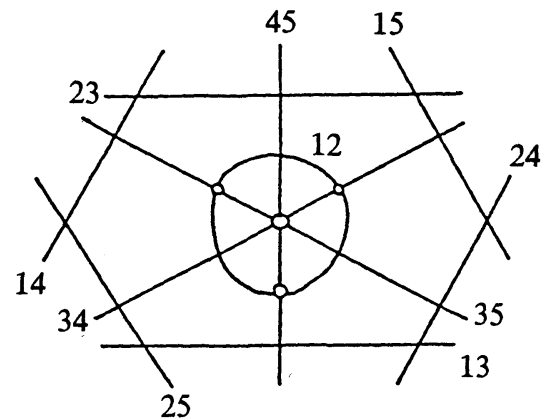

(i)

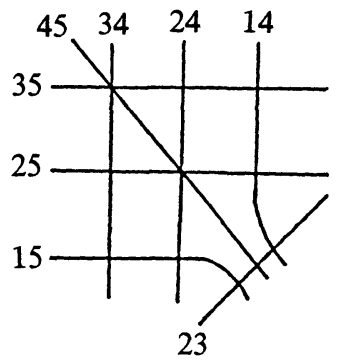

(iii)

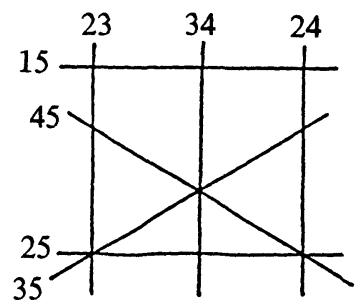

(v)

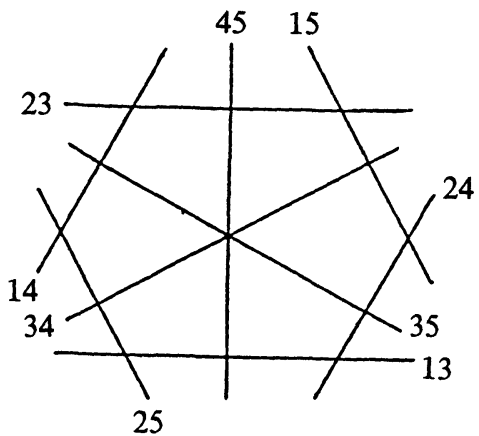

(ii)

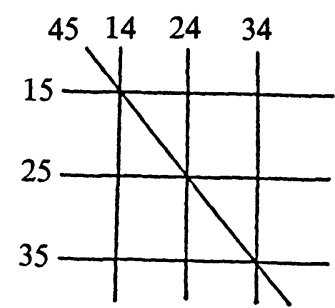

(iv)

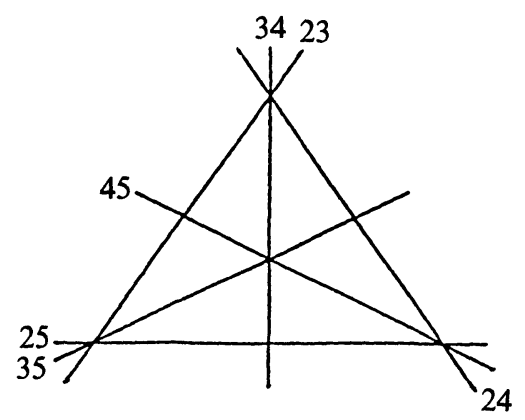

(vi)

FIGURE I (i)-(vi)

(In (i) open circles denote no intersection.)

Diagrams such as those in Figure I are not complete without specifying the self-intersection of the lines therein. Rather than labelling the lines in the diagrams, we do that here (with the same numbering):

(5.2)

(i) all lines -1

(ii) $34,35,45$ are 0 ; all others are -1 
(iii) 45 is $1 ; 14,15,23$ are -1 ; all others are 0

(iv) 45 is 2 ; all others are 0

(v) 15 is -1 ; 23, 2434 are 0 ; all others are 1

(vi) all lines 1.

REMARK 5.3. From these diagrams it is easy to see the blow-ups (vi) $\rightarrow$ (v) $\rightarrow$ (iii) $\rightarrow$ (i) $\rightarrow$ (i) (as well as (iv) $\rightarrow$ (iii))-compare 4.1.

REMARK 5.4. The configuration (vi) of lines in $\mathbf{P}^{2}$ is the configuration $A_{1}(6)$ of Hirzebruch $[\mathbf{H}]$. This configuration was used in $[\mathbf{H}]$ to produce examples of surfaces with $c_{1}^{2}=3 c_{2}$. If the technique of $[\mathbf{H}]$ is applied to configurations (i) - (v), exactly the same surfaces are obtained.

6. Geometric invariant theory. It was observed in [DM] 4.6 that the quotient $Q(\bar{\mu})$ has the following description in terms of Mumford's geometric invariant theory [MF].

For each $s \in S$ let $\pi_{s}: \mathbf{P}^{S} \rightarrow \mathbf{P}$ be the projection of $\mathbf{P}^{S}$ onto the $s$ th factor. Let $L_{s}$ be the pullback via $\pi_{s}$ of the tangent bundle $\mathcal{O}(2)$ on $\mathbf{P}$ and let

$$
L_{\underline{\mu}}=\bigotimes_{s \in S} L_{s}^{\otimes \mu_{s}} .
$$

This line bundle $L_{\mu}$ admits a natural $\mathrm{PGL}_{2}$ action. The stable and semi-stable points of $\mathbf{P}^{S}$ defined at 1.2 are the same as those defined in [MF] for this action, and $Q(\underline{\mu})$ is the same as Mumford's projective "quotient" variety.

The associated variety $Q^{*}(\underline{\mu})$ can also be described as a quotient as follows.

For each partition $\left\{S_{1}, S_{2}\right\}$ of $S$ with $\mu\left(S_{1}\right)=\mu\left(S_{2}\right)=d / 2$, let $Z_{\left\{S_{1}, S_{2}\right\}}$ be the subvariety of $M_{\text {sst }}$ consisting of all $\gamma \in M_{\text {sst }}$ such that $\gamma$ is constant on both $S_{1}$ and $S_{2}$. Then the $Z_{\left\{S_{1}, S_{2}\right\}}$ are disjoint nonsingular closed subvarieties of $M_{\text {sst }}$. Let $Z$ be their union and let $\rho: M^{*} \rightarrow M_{\text {sst }}$ be the blow-up of $M_{\text {sst }}$ along $Z$. Let $M_{\text {sst }}^{*}$ be the complement in $M^{*}$ of the proper transform of $M_{\text {cusp }}$ in $M^{*}$. The action of $\mathrm{PGL}_{2}$ on $M_{\text {stt }}$ induces an action on $M^{*}$ which preserves $M_{\mathrm{sst}}^{*}$.

LEMMA 6.1. The stabilizer in $\mathrm{PGL}_{2}$ of $\gamma \in M_{\mathrm{sst}}^{*}$ has order 2 if $\gamma \in \rho^{-1}(Z)$ and has order 1 otherwise.

Proof. The only element of $\mathrm{PGL}_{2}$ which fixes three distinct points of $\mathbf{P}$ is the identity. Hence $\mathrm{PGL}_{2}$ acts freely on $M_{\text {sst }}-Z$ and hence on $M_{\mathrm{sst}}^{*}-\rho^{-1}(Z)$. The stabilizer of any $\gamma \in Z$ is $\mathbf{C}^{*}$, and in an appropriate 
coordinate system this acts on the normal to $Z$ at $\gamma$ as

$$
\left(u_{1}, \ldots, u_{n-1}, v_{1}, \ldots, v_{N-n-1}\right) \rightarrow\left(t u_{1}, \ldots, t u_{n-1}, t^{-1} v_{1}, \ldots, t^{-1} v_{N-n-1}\right)
$$

where $\gamma \in Z_{\left\{S_{1}, S_{2}\right\}}$ and $n=c\left(S_{1}\right)$. In the associated projective representation the stabilizer of a point $(u, v)$ is $\{ \pm 1\}$ unless $u=0$ or $v=0$. In the latter cases $(u, v)$ represents a point in the proper transform of $M_{\text {cusp. }}$. Therefore the stabilizer in $\mathrm{PGL}_{2}$ of each $\gamma \in \rho^{-1}(Z) \cap M_{\mathrm{sst}}^{*}$ has order 2.

LeMma 6.2. The quotient $\mathrm{PGL}_{2} \backslash M_{\mathrm{sst}}^{*}$ is the geometric invariant theoretic quotient of $M_{\mathrm{sst}}^{*}$ by $\mathrm{PGL}_{2}$ and is a projective variety. It is non-singular and is naturally isomorphic to the blow-up $\tilde{Q}^{*}(\underline{\mu})$ of $Q^{*}(\underline{\mu})$ at the nonsingular cusps.

Proof. This is a special case of $\left[\mathbf{K}_{2}, 6.9\right]$.

7. The computation of $H^{*}\left(Q^{*}(\underline{\mu})\right)$. In this section we shall consider only the case $k=\mathrm{C}$. Our aim is to compute the cohomology (except for the 2-torsion) of the complex variety $Q^{*}(\underline{\mu})$.

In the last section we gave a description of $Q^{*}(\underline{\mu})$ in terms of geometric invariant theory, and this means that we can use the general procedure described in $\left[\mathbf{K}_{1}\right]$ to investigate its cohomology. For the convenience of the reader, instead of describing the procedure of $\left[\mathbf{K}_{\mathbf{1}}\right]$ in general and then applying it to our special case, we shall analyze the special case directly without overt reference to $\left[\mathbf{K}_{\mathbf{1}}\right]$.

Let $\mathbf{F}$ be any field of characteristic different from 2. For any topological space $A$ let $P(A)$ denote the Poincaré series of $A$ with coefficients in $\mathbf{F}$. That is,

$$
P(A)=\sum_{i \geq 0} t^{i} \operatorname{dim}_{\mathbf{F}} H^{i}(A ; \mathbf{F}) .
$$

If a group $G$ acts on $A$ let $P^{G}(A)$ denote the equivariant Poincaré series

$$
P^{G}(A)=\sum_{i \geq 0} t^{i} \operatorname{dim}_{\mathrm{F}} H_{G}^{i}(A ; \mathbf{F})
$$

where $H_{G}^{i}$ denotes equivariant cohomology (cf. $[\mathbf{A B}] \S 1$ or $\left.\left[\mathbf{K}_{1}\right] \S 2\right)$.

Throughout this section the group $\mathrm{PGL}_{2}$ will be denoted by $G$, and all cohomology will be with coefficients in $\mathbf{F}$.

By Lemma 6.2 the blow-up $\tilde{Q}^{*}(\underline{\mu})$ of $Q^{*}(\underline{\mu})$ at all the nonsingular cusps is isomorphic to $G \backslash M_{\mathrm{sst}}^{*}$. Therefore

$$
P\left(Q^{*}(\underline{\mu})\right)=P\left(G \backslash M_{\mathrm{sst}}^{*}\right)-m\left(t^{2}+\cdots+t^{2(N-4)}\right)
$$


where $m$ is the number of nonsingular cusps. By Lemma 6.1 the order of the stabilizer in $G$ of every point of $M_{\mathrm{sst}}^{*}$ is either 1 or 2 . This implies that

$$
P\left(G \backslash M_{\mathrm{sst}}^{*}\right)=P^{G}\left(M_{\mathrm{sst}}^{*}\right)
$$

since the characteristic of $\mathbf{F}$ is different from 2 (cf. [ $\left.\mathbf{K}_{\mathbf{1}}\right], 5.6$ and 8.14).

Recall that $M^{*}$ is the blow-up of $M_{\text {sst }}$ along $Z$ and that $M_{\text {sst }}^{*}$ is the complement in $M^{*}$ of the proper transform of $M_{\text {cusp. }}$. A point $\gamma \in M_{\text {sst }}$ belongs to $M_{\text {cusp }}$ if and only if there exists some $S_{1} \subseteq S$ with $\mu\left(S_{1}\right)=d / 2$ such that $\gamma$ is constant on $S_{1}$. The proper transform of $M_{\text {cusp }}$ in $M^{*}$ is the disjoint union of the proper transforms, $T\left(S_{1}\right)$ say, of the sets

$$
\left\{\gamma \mid \gamma \text { constant on } S_{1}\right\}
$$

for $S_{1} \subseteq S$ with $\mu\left(S_{1}\right)=d / 2$.

Each $T\left(S_{1}\right)$ is nonsingular and has codimension $c\left(S_{1}\right)-1$ in $M^{*}$. Let $B$ be the Borel subgroup of $G=\mathrm{PGL}_{2}$ represented by upper triangular matrices. Then

$$
T\left(S_{1}\right) \cong G \times_{B} Y\left(S_{1}\right)
$$

where $Y\left(S_{1}\right)$ is the proper transform of the set of $\gamma \in M_{\text {sst }}$ such that $\gamma(s)=\infty$ for each $s \in S_{1}$. This implies (see $[\mathbf{A B}] \S 13$ ) that

$$
H_{G}^{*}\left(T\left(S_{1}\right)\right) \cong H_{B}^{*}\left(Y\left(S_{1}\right)\right) \text {. }
$$

However, $B$ is homotopy equivalent to its maximal reductive subgroup $\mathrm{C}^{*}$, so

$$
H_{B}^{*}\left(Y\left(S_{1}\right)\right) \cong H_{\mathbf{C}^{*}}^{*}\left(Y\left(S_{1}\right)\right) .
$$

Moreover, $Y\left(S_{1}\right)$ retracts onto its intersection $Z\left(S_{1}\right)$ with the exceptional divisor $\rho^{-1}(Z)$ in $M^{*}$, and $Z\left(S_{1}\right)$ is isomorphic to projective space of dimension $N-c\left(S_{1}\right)-2$ and is fixed by $\mathbf{C}^{*}$. Therefore

$$
H_{\mathbf{C}^{*}}^{*}\left(Y\left(S_{1}\right)\right) \cong H_{\mathbf{C}^{*}}^{*}\left(Z\left(S^{1}\right)\right) \cong H^{*}\left(Z\left(S_{1}\right)\right) \times H^{*}\left(B \mathbf{C}^{*}\right) .
$$

The representation of $\mathbf{C}^{*}$ on the normal to $Y\left(S_{1}\right)$ (and hence a fortiori on the normal to $\left.T\left(S_{1}\right)\right)$ at any point of $Z\left(S_{1}\right)$ is scalar multiplication by the character $t \rightarrow t^{2}$.

The subvarieties $\left\{T\left(S_{1}\right) \mid S_{1} \subseteq S, \mu\left(S_{1}\right)=d / 2\right\}$ together with $M_{\text {sst }}^{*}$ form a smooth stratification of $M^{*}$. Since

$$
H_{G}^{*}\left(T\left(S_{1}\right)\right) \cong H_{\mathbf{C}^{*}}^{*}\left(Z\left(S_{1}\right)\right)
$$

the fact that $\mathrm{C}^{*}$ fixes $Z\left(S_{1}\right)$ pointwise and that its representation on the normal to $T\left(S_{1}\right)$ at any point of $Z\left(S_{1}\right)$ is primitive for any odd prime implies that this stratification is equivariantly perfect over the field $\mathbf{F}$ of 
characteristic $\neq 2$ ([AB] 1.9 and 13.4). In other words its equivariant Morse inequalities are all equalities, or equivalently

$$
P^{G}\left(M^{*}\right)=P^{G}\left(M_{\text {sst }}^{*}\right)+\sum t^{2\left(c\left(S_{1}\right)-1\right)} P^{G}\left(T\left(S_{1}\right)\right)
$$

where the sum is over all subsets $S_{1} \subseteq S$ satisfying $\mu\left(S_{1}\right)=d / 2$. By (7.5) and (7.6) this implies that

$$
P^{G}\left(M_{\mathrm{sst}}^{*}\right)=P^{G}\left(M^{*}\right)-\sum t^{2\left(c\left(S_{1}\right)-1\right)} P\left(\mathbf{P}^{N-c\left(S_{1}\right)-2}\right)\left(1-t^{2}\right)^{-1} .
$$

Now $M^{*}$ is the blow-up of $M_{\text {sst }}$ along the disjoint union of the subvarieties

$$
Z_{\left\{S_{1}, S_{2}\right\}}=\left\{\gamma \in M_{\text {sst }} \mid \gamma \text { constant on } S_{1} \text { and } S_{2}\right\}
$$

for all partitions $\left\{S_{1}, S_{2}\right\}$ of $S$ such that $\mu\left(S_{1}\right)=d / 2$. Each $Z_{\left\{S_{1}, S_{2}\right\}}$ is isomorphic to $C^{*} \backslash G$ and so its equivariant Poincaré series is $P\left(B C^{*}\right)=$ $\left(1-t^{2}\right)^{-1}$. Therefore

$$
P^{G}\left(M^{*}\right)=P^{G}\left(M_{\text {sst }}\right)+\sum\left(t^{2}+\cdots+t^{2(N-3)}\right)\left(1-t^{2}\right)^{-1}
$$

where the sum is over all partitions $\left\{S_{1}, S_{2}\right\}$ with $\mu\left(S_{1}\right)=d / 2$.

It is easy to check that

$$
\begin{aligned}
& \left(1-t^{2}\right)^{-1}\left(P\left(\mathbf{P}^{N-3}\right)-t^{2\left(c\left(S_{1}\right)-1\right)} P\left(\mathbf{P}^{N-c\left(S_{1}\right)-2}\right)-t^{2\left(c\left(S_{1}\right)-1\right)} P\left(\mathbf{P}^{N-c\left(S_{2}\right)-2}\right)\right) \\
& \quad=P\left(\mathbf{P}^{c\left(S_{1}\right)-2} \times \mathbf{P}^{c\left(S_{2}\right)-2}\right) .
\end{aligned}
$$

Therefore from (7.8) and (7.9) we obtain

$$
\text { (7.10) } \quad P^{G}\left(M_{\mathrm{sst}}^{*}\right)=P^{G}\left(M_{\mathrm{sst}}\right)+\sum\left[P\left(\mathbf{P}^{c\left(S_{1}\right)-2} \times \mathbf{P}^{c\left(S_{2}\right)-2}\right)-\left(1-t^{2}\right)^{-1}\right]
$$

where the sum is over all partitions $\left\{S_{1}, S_{2}\right\}$ of $S$ with $\mu\left(S_{1}\right)=\mu\left(S_{2}\right)=$ $d / 2$.

Now for each $S_{1} \subseteq S$ with $\mu\left(S_{1}\right)>d / 2$, let $T\left(S_{1}\right)$ be the set of all $\gamma \in \mathbf{P}^{S}$ such that there exists $x \in \mathbf{P}$ satisfying $\gamma(s)=x$ for all $s \in S_{1}$. Then

$$
T\left(S_{1}\right) \cong G \times_{B} Y\left(S_{1}\right)
$$

where

$$
Y\left(S_{1}\right)=\left\{\gamma \in \mathbf{P}^{S} \mid \gamma(s)=\infty \text { iff } s \in S_{1}\right\} \cong \mathbf{C}^{N-c\left(S_{1}\right)}
$$

Thus $T\left(S_{1}\right)$ is nonsingular of codimension $c\left(S_{1}\right)-1$ in $\mathbf{P}^{S}$ and

$$
P^{G}\left(T\left(S_{1}\right)\right)=P^{B}\left(Y\left(S_{1}\right)\right)=P^{C^{*}}\left(Y\left(S_{1}\right)\right) .
$$

Moreover, $Y\left(S_{1}\right)$ retracts onto the point $\gamma$ given by $\gamma(s)=\infty$ if $s \in S_{1}$ and $\gamma(s)=0$ otherwise. This point is fixed by $\mathbf{C}^{*}$. The representation of $\mathrm{C}^{*}$ on the normal to $T\left(S_{1}\right)$ at $\gamma$ is primitive (this time for all primes). 
Thus the argument used above shows that $M_{\text {sst }}$ together with the subvarieties $\left\{T\left(S_{1}\right) \mid S_{1} \subseteq S, \mu\left(S_{1}\right)>d / 2\right\}$ form a smooth stratification of $\mathbf{P}^{S}$ which is equivariantly perfect. Hence

$$
P^{G}\left(M_{\mathrm{sst}}\right)=P^{G}\left(\mathbf{P}^{S}\right)-\sum t^{2\left(c\left(S_{1}\right)-1\right)}\left(1-t^{2}\right)^{-1}
$$

where the sum is over all $S_{1} \subseteq S$ with $\mu\left(S_{1}\right)>d / 2$.

Finally by Proposition 5.8 of $\left[\mathbf{K}_{2}\right]$ and the fact that $\mathrm{PGL}_{2}$ is homotopy equivalent to its maximal compact subgroup we have

$$
P^{G}\left(\mathbf{P}^{S}\right)=P\left(\mathbf{P}^{S}\right) P(B G)=\left(1-t^{2}\right)^{N}\left(1-t^{4}\right)^{-1} .
$$

From (7.1), (7.2), (7.10), (7.11) and (7.12) we obtain the formula

$$
\begin{aligned}
P\left(Q^{*}(\underline{\mu})\right)= & \left(1+t^{2}\right)^{N}\left(1-t^{4}\right)^{-1}-\sum_{u, n} t^{2\left(c\left(S_{1}\right)-1\right)}\left(1-t^{2}\right)^{-1} \\
& +\sum_{s}\left[P\left(\mathbf{P}^{c\left(S_{1}\right)-2} \times \mathbf{P}^{c\left(S_{2}\right)-2}\right)-\left(1-t^{2}\right)^{-1}\right]
\end{aligned}
$$

where the first sum is over the partitions $\left\{S_{1}, S_{2}\right\}$ of $S$ which define an unstable point $\left(\mu\left(S_{1}\right)>d / 2\right)$ or a nonsingular cusp $\left(\mu\left(S_{1}\right)=d / 2\right.$ and $\left.c\left(S_{1}\right)=2\right)$, and the last sum is over the partitions $\left\{S_{1}, S_{2}\right\}$ defining a singular cusp $\left(\underline{\mu}\left(S_{1}\right)=d / 2\right.$ and $\left.c\left(S_{1}\right)>2\right)$.

The reader may verify that the right hand side of (7.13) is a polynomial of degree $2(N-3)$ in $t$ which satisfies Poincaré duality.

THEOREM 7.14. Let $\underline{\mu}$ be of any sequence of weights satisfying 1.1. Then

(i) $H^{i}\left(Q^{*}(\mu)\right.$; Z) has no odd torsion, for any $i$;

(ii) $H^{i}\left(Q^{*}(\mu) ; \mathbf{Z}\left[\frac{1}{2}\right]\right)=0$ for $i$ odd;

(iii) $H^{2 i}\left(Q^{*}(\mu) ; \mathbf{Z}\left[\frac{1}{2}\right]\right)$ has rank $b_{2 i}$ for any $i$ where $b_{2 i}$ is the coefficient of $t^{2 i}$ in the right hand side of 7.13 ( see Table III);

(iv) In the Hodge decomposition of $H^{i}\left(Q^{*}(\underline{\mu}) ; \mathbf{C}\right)$, the $(p, q)$-cohomology $H^{p, q}=0$ unless $p=q$.

Proof. The fact that the formula 7.13 is valid for any field of characteristic different from 2 implies that $H^{*}\left(Q^{*}(\underline{\mu}) ; \mathbf{Z}\right)$ has no odd torsion. (ii) and (iii) follow immediately from 7.13, while (iv) follows from the last remark of $\$ 14$ of $\left[K_{1}\right]$.

Using formula 7.13 it is then routine to compute the Poincare polynomials, and hence the even betti numbers $b_{2 i}$.

The answers are to be found in Table III, for all cases not covered by Theorem 4.1. (Recall that $Q\left(3^{6} 2\right)$ and $Q\left(772^{5}\right)$ are compact and hence non-singular; in all other cases $Q(\underline{\mu})$ is singular.) 
TABLE III

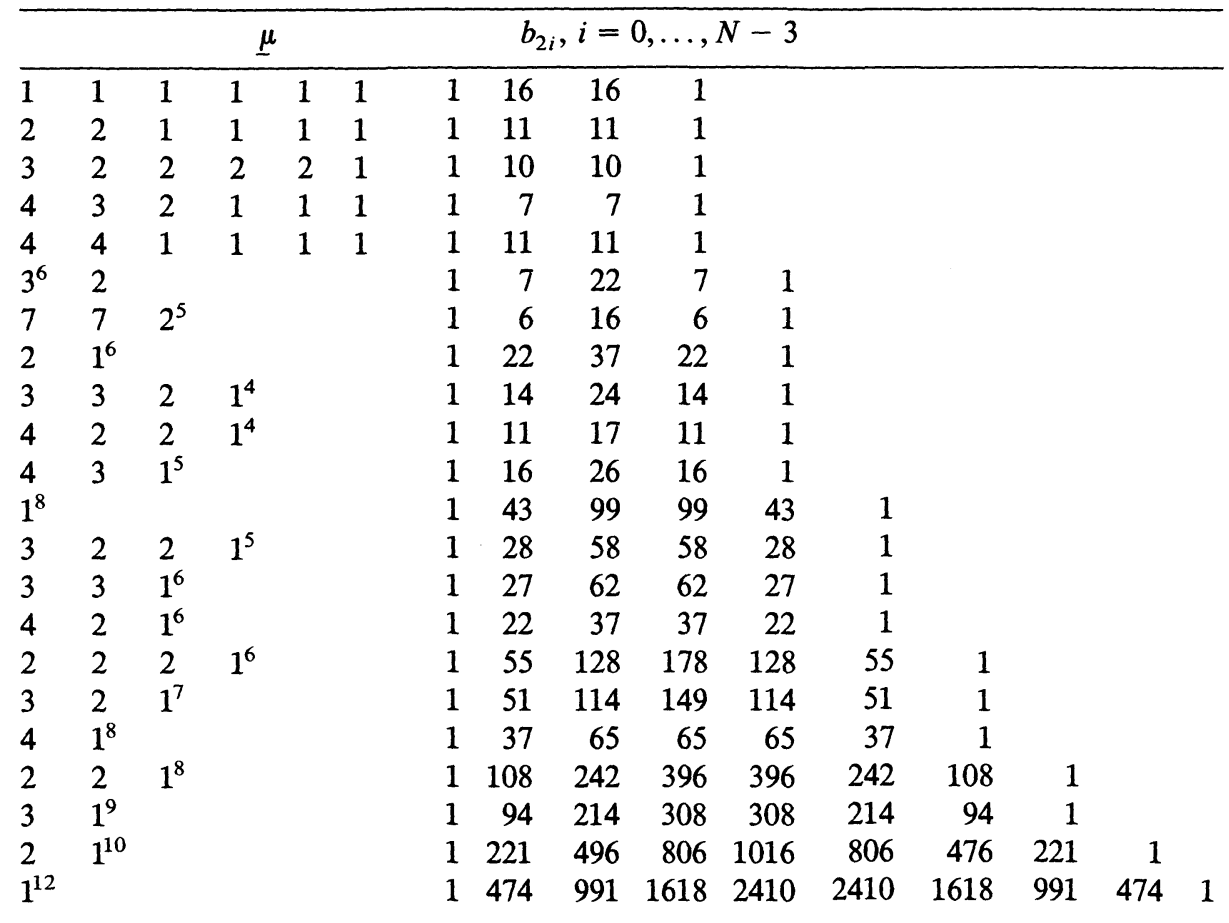

In the case $\underline{\mu}=1 \cdots 1$, the betti numbers were computed in $\left[\mathbf{K}_{\mathbf{1}}\right]$ in case $N$ odd (when there are no cusps) and in $\left[\mathbf{K}_{2}\right]$ in case $N$ even. The answer is

$$
\begin{aligned}
& N \text { odd: } b_{2 i}=\sum_{0}^{i}\left(\begin{array}{c}
N-1 \\
k
\end{array}\right), \quad i \leq(N-3) / 2, \\
& N \text { even: } b_{2 i}=\sum_{k=0}^{i}\left(\begin{array}{c}
N-1 \\
k
\end{array}\right)+\frac{1}{2}\left(\begin{array}{c}
N \\
N / 2
\end{array}\right)(i), \quad i \leq(N-4) / 2 .
\end{aligned}
$$

As a practical matter, in using 7.13 , it is only necessary to compute $P\left(Q^{*}(\underline{\mu})\right)$ up through dimension $N-3$, as Poincaré duality then yields the remaining terms.

REMARK 7.15. By (1.11), $Q^{*}(\mu)$ is rational. It is then a classical fact that $Q^{*}(\mu)$ is simply-connected and it is shown in [AM] that $H^{3}\left(Q^{*}(\underline{\mu}) ; \mathbf{Z}\right)$ is torsion-free (and hence, when $Q^{*}(\underline{\mu})$ is a three-fold $H^{i}\left(Q^{*}(\underline{\mu}) ; \mathrm{Z}\right)$ is torsion-free for all $i$.)

REMARK 7.16. If a finite group $G$ acts on a space $X$ and if $\mathbf{F}$ is a field of characteristic prime to the order of $G$, then $H^{*}(X / G ; \mathbf{F})=H^{*}(X ; \mathbf{F})^{G}$, the subspace of elements fixed by $G$. Thus the formulas of this section 
may be used to compute the cohomology of $Q_{\Sigma}^{*}(\mu)$ if the Poincaré polynomials therein are replaced by the Poincare polynomials of the fixed cohomology under the action of $\Sigma$, and the summations in (7.7)-(7.13) over various subsets of $\{1, \ldots, N\}$ are taken over various $\Sigma$-orbits instead. We leave these computations to the reader, except for the following ones (which are the only complicated cases in which $Q_{\Sigma}(\underline{\mu})$ is a $V$-manifold):

(7.17) If $\underline{\mu}=3^{6} 2$ (and $\Sigma=\Sigma_{6}$ ) or $\underline{\mu}=772^{5}$ (and $\Sigma=\Sigma_{5}$ ) then the even betti numbers of $Q_{\Sigma}(\underline{\mu})$ are $b_{2 i}=\overline{1}, 2,3,2,1$ for $i=0, \ldots, 4$.

8. Intersection homology. It is also possible to calculate the dimension of the rational intersection homology (with respect to the middle perversity) groups of the singular variety $Q(\underline{\mu})$. Of course this is the same as calculating the dimension of the corresponding rational intersection cohomology groups $I H^{i}(Q(\mu))$.

Throughout this section, all (co)homology, singular or intersection, is to be taken with rational coefficients.

Let $E$ be the exceptional divisor of the blow-up $\pi: Q^{*}(\underline{\mu}) \rightarrow Q(\underline{\mu})$. Let $\tilde{U}$ be an open neighborhood of $E$ in $Q^{*}(\mu)$ which is isomorphic to the normal bundle to $E$ in $Q^{*}(\underline{\mu})$. Let $U=\pi(\tilde{U})$ which we may assume to be a union of disjoint open contractible neighborhoods of the singular cusps in $Q(\underline{\mu})$.

Since $\tilde{U}$ is nonsingular we have

$$
I H^{i}(\tilde{U}) \cong H^{i}(\tilde{U}) \cong H^{i}(E) \text { for all } i .
$$

Since $U$ has isolated singularities and dimension $N-3$ we have

$$
I H^{i}(U) \cong H^{i}(U)=0 \text { for } i>N-3
$$

and

$$
I H^{N-3}(U) \cong \operatorname{Im}\left(H^{N-3}\left(U-Q_{\text {s cusp }}\right) \rightarrow H^{N-3}(U)\right)=0
$$

where $Q_{\text {s cusp }}$ is the set of singular cusps in $Q(\underline{\mu})$ ([CGM, 2.28]).

By [CGM, 5.1] there are natural embeddings

$$
I H_{i}(Q(\underline{\mu})) \rightarrow I H_{i}\left(Q^{*}(\underline{\mu})\right)
$$

for $i \leq N-3$. These induce surjective maps

$$
I H^{i}\left(Q^{*}(\underline{\mu})\right) \rightarrow H^{i}(Q(\underline{\mu}))
$$

for $i \leq N-3$, and hence by Poincaré duality embeddings

$$
I H^{i}(Q(\underline{\mu})) \rightarrow I H^{i}\left(Q^{*}(\underline{\mu})\right)
$$

for $i \geq N-3$. 
Since $\tilde{U} \cap \rho^{-1}\left(Q_{\text {st }}\right)$ is isomorphic to $U \cap Q_{\text {st }}$ and is nonsingular we have Mayer-Vietoris sequences for ordinary and intersection cohomology as follows for $i \geq N-3$ :

$\rightarrow H^{i-1}\left(U \cap Q_{\mathrm{st}}\right) \rightarrow H^{i}\left(Q^{*}(\underline{\mu})\right) \rightarrow H^{i}(\tilde{U})+H^{i}\left(Q_{\mathrm{st}}\right) \quad \rightarrow \quad H^{i}\left(U \cap Q_{\mathrm{st}}\right) \rightarrow$

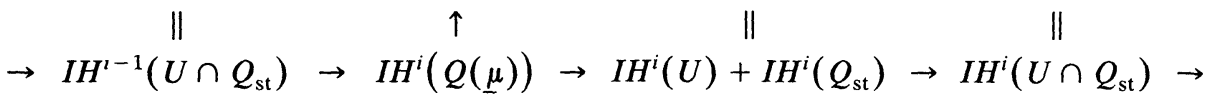
$\|$

0

From this it follows that

(8.1) $\operatorname{dim} I H^{i}(Q(\underline{\mu}))=\operatorname{dim} H^{i}\left(Q^{*}(\underline{\mu})\right)-\operatorname{dim} H^{i}(\tilde{U})+\operatorname{dim} I H^{i}(U)$

$$
=\operatorname{dim} H^{i}\left(Q^{*}(\underline{\mu})\right)-\operatorname{dim} H^{i}(E)
$$

for $i \geq N-3$.

By 1.10 the exceptional divisor $E$ is the disjoint union over partitions $\left\{S_{1}, S_{2}\right\}$ of $S$ with $\mu\left(S_{1}\right)=d / 2$ and $c\left(S_{1}\right)>2$ of $\mathbf{P}^{c\left(S_{1}\right)-2} \times \mathbf{P}^{c\left(S_{2}\right)-2}$. Therefore for $i \geq N-3$ the dimension of $I H^{i}(Q(\underline{\mu}))$ is equal to the coefficient of $t^{i}$ in the series

$$
\begin{aligned}
& P\left(Q^{*}(\underline{\mu})\right)-\sum_{s} P\left(\mathbf{P}^{c\left(S_{1}\right)-2} \times \mathbf{P}^{c\left(S_{2}\right)-2}\right) \\
& \quad=\left(1+t^{2}\right)^{N}\left(1-t^{4}\right)^{-1}-\sum_{u, n} t^{2\left(c\left(S_{1}\right)-1\right)}\left(1-t^{2}\right)^{-1}-\sum_{s}\left(1-t^{2}\right)^{-1}
\end{aligned}
$$

with the same conventions as before for the sums $\sum_{s}$ and $\sum_{u, n}$. If $i$ is odd this coefficient is zero, and if $i$ is even it is

$$
\begin{gathered}
\sum_{j \leq i / 2}\left(\begin{array}{c}
N-1 \\
j
\end{array}\right)-c\left\{\text { partitions }\left\{S_{1}, S_{2}\right\} \text { of } S \mid \underline{\mu}\left(S_{1}\right)>d / 2,\right. \\
\left.c\left(S_{1}\right) \leq i / 2+1\right\} \\
-c\left\{\text { partitions }\left\{S_{1}, S_{2}\right\} \text { of } S\left\lfloor\underline{\mu}\left(S_{1}\right)=d / 2\right\} .\right.
\end{gathered}
$$

It follows by Poincaré duality that if $i \leq N-3$ then

$$
\begin{aligned}
& \operatorname{dim} I H^{i}(Q(\underline{\mu}))=\operatorname{dim} I H^{2(N-3)-i}(Q(\underline{\mu})) \\
& =\sum_{j \leq N-3-i / 2}\left(\begin{array}{c}
N-1 \\
j
\end{array}\right)-c\left\{\text { partitions }\left\{S_{1}, S_{2}\right\} \text { of } S \mid \underline{\mu}\left(S_{1}\right)>d / 2\right. \\
& \left.\quad c\left(S_{1}\right) \leq N-i / 2-2\right\} \\
& \quad-c\left\{\text { partitions }\left\{S_{1}, S_{2}\right\} \text { of } S \mid \underline{\mu}(S)=d / 2\right\}
\end{aligned}
$$


Alternatively using Poincaré duality for $Q^{*}(\underline{\mu})$ and $E$ we have the formula

$$
\operatorname{dim} I H^{i}(Q(\underline{\mu}))=\operatorname{dim} H^{i}\left(Q^{*}(\underline{\mu})\right)-\operatorname{dim} H^{i-2}(E)
$$

for $i \leq N-3$. From this we see that if $i \leq N-3$ then the dimension of $I H^{i}(Q(\underline{\mu}))$ is equal to coefficient of $t^{i}$ in the series

$$
\begin{aligned}
P\left(Q^{*}(\underline{\mu})\right)-\sum_{s} t^{2} P\left(\mathbf{P}^{c\left(S_{1}\right)-2} \times \mathbf{P}^{c\left(S_{2}\right)-2}\right) \\
=\left(1+t^{2}\right)^{N}\left(1-t^{4}\right)^{-1}-\sum_{u, n} t^{2\left(c\left(S_{1}\right)-1\right)}\left(1-t^{2}\right)^{-1} \\
\quad+\sum_{s}\left[\left(1-t^{2}\right) P\left(\mathbf{P}^{c\left(S_{1}\right)-2} \times \mathbf{P}^{c\left(S_{2}\right)-2}\right)-\left(1-t^{2}\right)^{-1}\right] .
\end{aligned}
$$

When $i \leq N-3$ is even this coefficient is given by

$$
\begin{array}{r}
\sum_{j \leq i / 2}\left(\begin{array}{c}
N-1 \\
j
\end{array}\right)-c\left\{\text { partitions }\left\{S_{1}, S_{2}\right\} \text { of } S \mid \underline{\mu}\left(S_{1}\right) \geq d / 2,\right. \\
\left.c\left(S_{1}\right) \leq i / 2+1\right\} .
\end{array}
$$

The reader can check that the two formulas 8.3 and 8.5 do indeed agree.

Thus we have proved

THEOREM 8.6. The dimension of the ith intersection homology group of $Q(\underline{\mu})$ is 0 if $i$ is odd and if $i$ is even it is

$$
\begin{array}{r}
\sum_{j \leq \min (i / 2, N-3-i / 2)}\left(\begin{array}{c}
N-1 \\
j
\end{array}\right)-c\left\{\text { partitions }\left\{S_{1}, S_{2}\right\} \text { of } S \mid \underline{\mu}\left(S_{1}\right) \geq d / 2,\right. \\
\left.c\left(S_{1}\right) \leq \min (i / 2, N-3-i / 2)+1\right\} .
\end{array}
$$

REMARK 8.7. This computation is a special case of a general procedure for computing the intersection Betti numbers of the geometric invariant theoretic quotient of a nonsingular complex projective variety by a reductive group action described in $\left[\mathbf{K}_{\mathbf{3}}\right]$.

9. Algebraic cycles in $Q^{*}(\underline{\mu})$. Let us fix $\underline{\mu}$, set $Q^{*}=Q^{*}(\underline{\mu})$, and let $D=Q^{*}-Q$. Then $D$ is a union of components of complex dimension $N-4$.

THEOREM 9.1. Let $\mathbf{F}$ be a field of characteristic not equal to 2. Then the inclusion $D \rightarrow Q^{*}$ induces epimorphisms on homology with coefficients in $\mathbf{F}$ in dimension less than or equal to $N-4$ and in dimension $2(N-4)$ ( = codimension 2$)$. 
Proof. We have the exact sequence of the pair

$$
H_{i+1}\left(Q^{*}, D\right) \rightarrow H_{i}(D) \rightarrow H_{i}\left(Q^{*}\right) \rightarrow H_{i}\left(Q^{*}, D\right)
$$

and the equality $H_{i}\left(Q^{*}, D\right)=H^{2(N-3)-i}(Q)$ from Alexander duality.

By the argument of $\left[\mathbf{L W}_{2}, 4.15\right] H^{i}(Q)$ vanishes for $i>N-3$, yielding the first part of theorem, and $H^{1}(Q)$ has rank $\left(\begin{array}{c}N-2 \\ 2\end{array}\right)-1$.

For the second part of the theorem, the relevant part of the exact sequence is

$$
H^{1}(Q) \rightarrow H_{2(N-4)}(D) \rightarrow H_{2(N-4)}\left(Q^{*}\right)
$$

so we need only show

$$
\begin{aligned}
\operatorname{rank}\left(H_{2(N-4)}(D)\right) & =\operatorname{rank}\left(H_{2(N-4)}\left(Q^{*}\right)\right)+\operatorname{rank}\left(H^{1}(Q)\right) \\
& =\operatorname{rank}\left(H^{2}\left(Q^{*}\right)\right)+\left(\begin{array}{c}
N-2 \\
2
\end{array}\right)-1
\end{aligned}
$$

by Poincaré duality.

Let us examine the terms in the expression for $P\left(Q^{*}\right)$ up through dimension 2. We see

$$
P\left(Q^{*}\right)=\left(1-t^{2}\right)^{-1}\left[\left(1+t^{2}\right)^{N-1}-\sum_{u, n}^{\prime} t^{2}+\sum_{s}\left[\left(1+t^{2}\right)-1\right]\right]+\cdots
$$

where $\sum_{u, n}^{\prime}$ is taken over all unstable or semi-stable pairs (i.e. all $\{i, j\}$ with $\mu_{i}+\mu_{j} \geq d / 2$ ) and $\Sigma_{s}$ is taken over all singular cusps (determined by subsets $S_{1}$ of $S$ with $\left.\mu\left(S_{1}\right)=d / 2, c\left(S_{1}\right) \geq 3\right)$.

This gives the expression for the second betti number

$$
b_{2}=1+(N-1)-c_{1}+c_{2}
$$

where $c_{1}$ is the number of summands in $\Sigma_{u, n}^{\prime}$ and $c_{2}$ the number of summands in $\Sigma_{s}$.

Then there are $\left(\begin{array}{l}N \\ 2\end{array}\right)-c_{1}$ pairs $\{i, j\}$ with $\mu_{i}+\mu_{j}<d / 2$. Thus $D$ has $\left(\begin{array}{l}N \\ 2\end{array}\right)-c_{1}+c_{2}$ components, so we obtain

$$
\left(\begin{array}{c}
N \\
2
\end{array}\right)-c_{1}+c_{2}=b_{2}-N+\left(\begin{array}{c}
N \\
2
\end{array}\right)=b_{2}+\left(\begin{array}{c}
N-2 \\
2
\end{array}\right)-1
$$

as required.

Conjecture 9.2. The inclusion $D \rightarrow Q^{*}$ (or $D \rightarrow Q_{\Sigma}^{*}$ ) induces epimorphisms in homology in all dimensions $\leq 2(N-4)$.

10. Group cohomology. In this section we consider the rational cohomology of the groups $\Gamma(\underline{\mu})$ in the case where $Q(\underline{\mu})$ is either compact or has only nonsingular cusps, and in the additional case $\underline{\mu}=111111$, where we use an entirely different method. 
In any case, $H^{*}(\Gamma(\underline{\mu}))=H^{*}\left(Q_{\text {st }}(\underline{\mu})\right)$. However, if $Q(\underline{\mu})$ is compact then $H^{*}(\Gamma(\underline{\mu}))=H^{*}(Q(\underline{\mu}))$, whereas if $Q(\underline{\mu})$ has $\infty_{n}$ nonsingular cusps, $H^{*}(\Gamma(\underline{\mu}))=H^{*}\left(Q(\underline{\mu})-\infty_{n}\right.$ points $)$.

The cohomology then follows immediately from Table I, and Theorem 4.1 , in all cases except $\mu=3^{6} 2$ or $772^{5}$, and these last two are covered by Table III. Similarly, when $Q(\underline{\mu})$ is non-singular, we may compute the rational cohomology of $\Gamma_{\Sigma}(\underline{\mu})$ by using (7.15).

We now specialize to the case of $\mu=1111111$. Then the space $Q^{*}(\mu)$ has been extensively studied under a different guise-as the Igusa compactification of the Siegel space of degree two and level two [G], $\left[\mathbf{L} \mathbf{W}_{1}\right],\left[\mathbf{L} \mathbf{W}_{2}\right]$. We shall use the work of $\left[\mathbf{L W}_{2}\right]$ to get some finer information, and in particular to determine the homology of $Q_{\mathrm{st}}\left(\begin{array}{llllll}1 & 1 & 1 & 1 & 1 & 1\end{array}\right)$.

THEOREM 10.1. Let $\mathbf{F}$ be a field of characteristic not equal to 2. Then

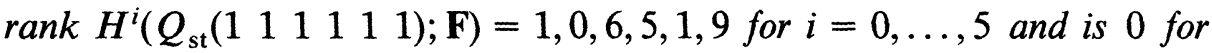
$i>5$.

Proof. Let $D=Q^{*}-Q$, and $C=Q^{*}-Q_{\mathrm{st}}$. We consider the exact sequence of the pair $\left(Q^{*}, C\right)$. Let us recall the results of $\left[\mathbf{L W}_{\mathbf{2}}\right]$ :

In $\left[\mathbf{L W} \mathbf{W}_{2}\right] C$ was called the union of the Humbert surfaces and $D$ was called the union of the boundary and Humbert surfaces. The group $\Sigma_{6}$, the symmetric group on six elements, acts on $Q^{*}$ by permuting the coordinates. If we let $\left\{S_{1}, S_{2}\right\}$ be partitions of $\{1, \ldots, 6\}$ with $\mu\left(S_{i}\right)=$ $c\left(S_{i}\right)=3$, the components of $C$ are indexed by such partitions. Letting $\Delta_{0}=\{\{1,2,3\},\{4,5,6\}\}$ and denoting the corresponding component of $C$ by $C_{0}$, then using the results of $\left[\mathbf{L W}_{\mathbf{2}}\right]$ we have, in the interesting dimensions 2 and 4:

$$
\begin{gathered}
\quad H_{4}(C) \rightarrow H_{4}\left(Q^{*}\right), \quad H_{2}(C) \rightarrow H_{2}\left(Q^{*}\right) \\
\operatorname{Ind}_{P}(\tau) \rightarrow \operatorname{Ind}_{P}(\tau)+F, \quad \operatorname{Ind}_{P}(\tau+\sigma) \rightarrow \operatorname{Ind}_{P}(\tau)=\tau+F .
\end{gathered}
$$

Here $\tau$ denotes the trivial representation, Ind $_{P}$ the induced representation from $P$ (the stabilizer of $\Delta_{0}$ ) to $\Sigma_{6}$, and $\sigma$ the one-dimensional representation in which the permutation $(14)(23)(56)$ is multiplication by -1. (This gives the action of $P$ on $H_{2}\left(C_{0}\right)$-this element switches the two factors in $C_{0}=\mathbf{P}^{1} \times \mathbf{P}^{1}$. The representation $F$ is the representation of the same name in $\left[\mathbf{L W _ { 2 }}\right], 3.1 .5$.)

We first show that the inclusion of each of the representations Ind $_{P}(\tau)$ is a monomorphism. Consider the map on $H_{4}$. For each partition $\Delta=\left\{\{i, j, k\},\left\{i^{\prime}, j^{\prime}, k^{\prime}\right\}\right\}$ let $t_{\Delta}$ be the intersection of the component $C_{\Delta}$ with the union of the components $\Delta_{i j}$ and $\Delta_{i^{\prime}, j^{\prime}}$, so $t_{0}$ represents the 
component of $H_{2}\left(C_{0}\right)$ acted trivially on by $P$. The intersection number of the component $C_{D}$ with $t_{\Delta}$ is the sum of the self-intersections of $\left(C_{\Delta} \cap \Delta_{i j}\right)$ in $\Delta_{i j}$ and $\left(C_{\Delta} \cap \Delta_{i^{\prime} j^{\prime}}\right)$ in $\Delta_{i^{\prime} j^{\prime}}$, which is $-1+-1=-2$ by [ $\left.\mathbf{L W}_{\mathbf{2}}\right]$, 2.3.4. Since the different components of $C$ are disjoint, this gives a pairing of the two copies of $\operatorname{Ind}_{P}(\tau)$ which has determinant $(-2)^{10}$ and is hence nonsingular.

Hence it remains to determine the map $\operatorname{Ind}_{P}(\sigma) \rightarrow \tau+F$. An easy calculation (using Frobenius reciprocity) shows $\operatorname{Ind}_{P}(\sigma)$ does not contain $\tau$, so it remains to determine whether the map to $F$ has non-zero image (as it will then be onto $F$, since $F$ is irreducible). As $F$ is self-dual we instead evaluate the image against $F$. A typical element of $F$ is $f=$ $3(\{1,2\}+\{1,3\}+\{1,4\}+\{1,5\}+\{1,6\})-\Sigma\{i, j\}$. If $s_{0}$ represents a generator of the representation $\sigma$, we may take $s_{0}=\left(C_{0} \cap \Delta_{12}\right)-\left(C_{0} \cap\right.$ $\left.\Delta_{45}\right)$. Taking the intersection of $s_{0}$ with the sum of the components $\Delta_{i j}$ indexed by $f$, we find the answer is -2 . (Note $\left(C_{0} \cap \Delta_{12}\right) \cap \Delta_{12}=0$ as this is the self-intersection of $\left(C_{0} \cap \Delta_{12}\right)$ in $C_{0}=\mathbf{P}^{1} \times \mathbf{P}^{1}$, but $\left(C_{0} \cap \Delta_{45}\right)$ $\cap \Delta_{12}=1$ as this is a transverse intersection-in fact the intersection of the two generators of $H_{2}\left(C_{0}\right)$. Similarly for $\Delta_{13}$, and the intersection is empty for $\Delta_{14}, \Delta_{15}$, and $\Delta_{16}$.) The theorem then follows readily.

REMARK 10.2. By comparison with $\left[\mathbf{L} \mathbf{W}_{2}\right]$ we may identify

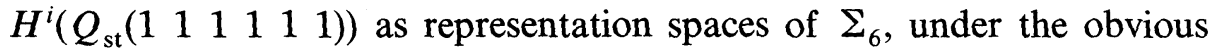
action. For $i=2,3,4,5$ they are respectively the representations with Young diagrams [6] + [51], [51], [6], [42].

\section{REFERENCES}

[AM] M. Artin and D. Mumford, Some elementary examples of unirational varieties which are not rational, Proc. London Math. Soc., 25 (1972), 75-95.

[AB] M. F. Atiyah and R. Bott, The Yang-Mills equations over Riemann surfaces, Phil. Trans. R. Soc. London, A 308 (1982), 523-615.

[CGM] J. Cheeger, M. Goresky and R. MacPherson, $L^{2}$-cohomology and intersection homology of singular algebraic varieties, in Seminar on Differential Geometry, S.-T. Yau, ed., Princeton Univ. Press, (1982), 303-340.

[DM] P. Deligne and G. D. Mostow, Monodromy of hypergeometric functions and non-integral lattice monodromy, Publ. Math. I.H.E.S., 63 (1986), 5-89.

[G] G. van der Geer, On the geometry of a Siegel modular threefold, Math. Ann., 260 (1982), 317-350.

[H] F. Hirzebruch, Arrangements of lines and algebraic surfaces, in Arithmetic and Geometry vol. II, M. Artin and J. Tate eds., Birkhauser, (1983), 113-140.

$\left[\mathrm{K}_{1}\right] \quad \mathrm{F}$. C. Kirwan, Cohomology of quotients in symplectic and algebraic geometry, Mathematical Notes series, Princeton University Press, 1985.

$\left[\mathrm{K}_{2}\right] \quad$ Partial desingularisations of quotients of nonsingular varieties and their Betti numbers, Ann. Math., 122 (1985), 41-85. 
$\left[\mathrm{K}_{3}\right] \_$, Rational intersection homology of quotients of nonsingular varieties, to appear.

$\left[\mathrm{LW}_{1}\right] \quad \mathrm{R}$. Lee and S. H. Weintraub, Cohomology of a Siegel modular variety of degree two, in Group Actions on Manifolds, R. Schultz ed., Amer. Math. Soc., Providence, (1985), 433-488.

$\left[\mathrm{LW}_{2}\right] \quad$, Cohomology of $\mathrm{Sp}_{4}(\mathrm{Z})$ and related groups and spaces, Topology, 24 (1985), 391-410.

[M] D. Mostow, Generalized Picard lattices arising from half-integral conditions, Publ. Math. I.H.E.S., 63 (1986), 91-106.

[MF] D. Mumford and J. Fogarty, Geometric Invariant Theory, Springer-Verlag, 1982.

Received February 12, 1986.

MATHEMATICAL INSTITUTE

OXFORD UNIVERSITY

24-29 ST. GILES

OXFORD, OX1 3LB

ENGLAND

DePARTMENT OF MATHEMATICS

YALE UNIVERSITY

BoX 2155 Yale Station

New Haven, CT 06520

USA

MATHEMATISCHES INSTITUT

UNIVERSITÄT GOTTINGEN

BUNSENSTRASSE 3-5

D-3400 GOTTINGEN

FEDERAL REPUBLIC OF GERMANY 



\section{PACIFIC JOURNAL OF MATHEMATICS EDITORS}

V. S. VARADARAJAN (Managing Editor) University of California Los Angeles, CA 90024 HERBERT ClEMENS University of Utah Salt Lake City, UT 84112

R. FINN

Stanford University

Stanford, CA 94305

\section{HERMANN FLASCHKA} University of Arizona Tucson, AZ 85721

RAMEsh A. GANGolli University of Washington Seattle, WA 98195

VAUghan F. R. JONES

University of California Berkeley, CA 94720

\section{ROBION KIRBY}

University of California

Berkeley, CA 94720

C. C. MOORE

University of California

Berkeley, CA 94720

HAROLD STARK

University of California, San Diego

La Jolla, CA 92093

\section{ASSOCIATE EDITORS}
R. ARENS
E. F. BECKENBACH
B. H. NEUMANN
F. WOLF
K. YOSHIDA (1906-1982)

\section{SUPPORTING INSTITUTIONS}

UNIVERSITY OF ARIZONA

UNIVERSITY OF BRITISH COLUMBIA

CALIFORNIA INSTITUTE OF TECHNOLOGY

UNIVERSITY OF CALIFORNIA

MONTANA STATE UNIVERSITY

UNIVERSITY OF NEVADA, RENO

NEW MEXICO STATE UNIVERSITY

OREGON STATE UNIVERSITY
UNIVERSITY OF OREGON

UNIVERSITY OF SOUTHERN CALIFORNIA

STANFORD UNIVERSITY

UNIVERSITY OF HAWAII

UNIVERSITY OF TOKYO

UNIVERSITY OF UTAH

WASHINGTON STATE UNIVERSITY

UNIVERSITY OF WASHINGTON 


\section{Pacific Journal of Mathematics}

Vol. 130, No. $1 \quad$ September, 1987

K. Adachi, Continuation of bounded holomorphic functions from certain subvarieties to weakly pseudoconvex domains $\ldots \ldots \ldots \ldots \ldots \ldots \ldots$

Erazm Jerzy Behr, Enveloping algebras of Lie superalgebras ........... 9

Dong M. Chung, Scale-invariant measurability in abstract Wiener spaces . . . 27

Peter Gerard Dodds and Bernardus de Pagter, Algebras of unbounded

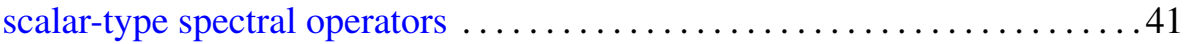

Wu-Yi Hsiang and Hsueh-Ling Huynh, Generalized rotational

hypersurfaces of constant mean curvature in the Euclidean spaces. II . . . .75

Harvey Bayard Keynes and M. Sears, Time changes for $\mathbf{R}^{n}$ flows and suspensions ..................................... 97

Frances Kirwan, Ronnie Lee and Steven Howard Weintraub, Quotients

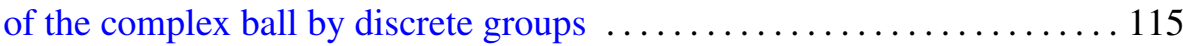

Magnhild Lien, Groups of knots in homology 3-spheres that are not

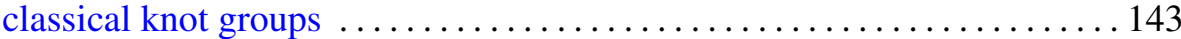

Juan Carlos Migliore, Liaison of a union of skew lines in $\mathbf{P}^{4} \ldots \ldots \ldots \ldots 153$

Jesper M. Møller, Spaces of sections of Eilenberg-Mac Lane fibrations . . . . 171 Daniel Ullman, A generalization of a theorem of Atkinson to noninvariant

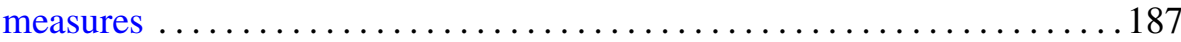

Kohhei Yamaguchi, Operations which detect $\mathscr{P}^{1}$ in odd primary connective

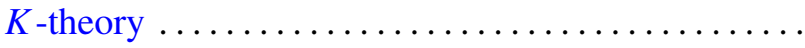

